\title{
Regularized Orthogonal Machine Learning for Nonlinear Semiparametric Models
}

\author{
Nekipelov, Denis ${ }^{\dagger}$ And Semenova, Vira ${ }^{\ddagger}$ And Syrgkanis, VAsilis ${ }^{\star 1}$ \\ ${ }^{\dagger} 254$ Monroe Hall University of Virginia, Charlottesville, VA 22904, USA \\ E-mail: denis@virginia.edu \\ ¥530 Evans Hall, University of California, Berkeley, CA 94720 \\ E-mail: semenovavira@gmail.com \\ * 1 Memorial Drive, Cambridge, MA, 02142 \\ E-mail: vasy@microsoft.com
}

\begin{abstract}
Summary This paper proposes a Lasso-type estimator for a high-dimensional sparse parameter identified by a single index conditional moment restriction (CMR). In addition to this parameter, the moment function can also depend on a nuisance function, such as the propensity score or the conditional choice probability, which we estimate by modern machine learning tools. We first adjust the moment function so that the gradient of the future loss function is insensitive (formally, Neyman-orthogonal) with respect to the first-stage regularization bias, preserving the single index property. We then take the loss function to be an indefinite integral of the adjusted moment function with respect to the single index. The proposed Lasso estimator converges at the oracle rate, where the oracle knows the nuisance function and solves only the parametric problem. We demonstrate our method by estimating the short-term heterogeneous impact of Connecticut's Jobs First welfare reform experiment on women's welfare participation decision.
\end{abstract}

Keywords: Conditional moment restrictions, single index models, high-dimensional sparse estimation, M-estimation, machine learning, Neyman-orthogonality

\section{INTRODUCTION}

Conditional moment restrictions (CMRs) often emerge as natural restrictions summarizing conditional independence, exclusion, or structural assumptions in economic models. In such a setting, a major challenge is to allow for a data-driven selection among a (very) large number of conditioning covariates. The sparsity assumption, which requires the number of relevant covariates to be small, has appeared to be an interpretable and plausible alternative. If the target parameter minimizes some population loss, a natural way to impose sparsity is to add an $\ell_{1}$-penalty on the target parameter to the sample loss. As discussed in Efron et al. (2007), this Lasso approach has substantial computational and statistical advantages over its alternatives. However, in general, the loss may be difficult to find. Focusing on a class of single index CMRs (Ichimura (1993), Klein and Spady (1993)), we make the Lasso approach feasible by deriving the loss for an arbitrary single index moment function and establish convergence rate for the Lasso estimator.

\footnotetext{
${ }^{1}$ We are extremely thankful to the editor and three anonymous referees for the comments that helped us improve the paper. We thank Victor Chernozhukov for many helpful discussions and pointing us to helpful references. We also thank Patrick Kline, Whitney Newey, James Powell and seminar participants at Harvard/MIT econometrics seminar and Microsoft Research for useful comments.
} 
Nekipelov et al.

The starting point of the analysis is a single index CMR that, in addition to target parameter, may depend on a nuisance component, such as the propensity score, the conditional choice probability, the conditional density, or alike, that can be substantially more high-dimensional than the target parameter itself. A natural approach would be to plug a machine learning/regularized estimate of the nuisance parameter into the moment function, such as random forest or neural networks. We first adjust the moment function so that the gradient of the future loss function is insensitive (i.e., Neyman-orthogonal, Chernozhukov et al. (2018)) with respect to the first-stage regularization bias, preserving the single index property. We then take the loss function to be an indefinite integral of the adjusted moment function with respect to the single-index. If the original moment function is monotone in the single-index, we report the global minimum of $\ell_{1}$ regularized $M$-estimator loss, following Negahban et al. (2012). Under mild conditions, the proposed estimator converges at the oracle rate, where the oracle knows the true value of the nuisance parameter and solves only the parametric problem.

We demonstrate the utility of our method with theoretical and empirical applications. First, we introduce a partially linear logistic model with heterogeneous treatment effects and derive an orthogonal loss. Second, we derive an orthogonal loss in conditional moment models with missing data, as studied in Carroll et al. (1995), Carroll and Wand (1991), Chen et al. (2008), Lee and Sepanski (1995), Sepanski and Carroll (1993) and static games of incomplete information (e.g. see Bajari et al. (2010) and Bajari et al. (2013) among others). In all these settings, we give sufficient primitive conditions on the nuisance parameters to achieve oracle convergence. In the empirical application, we study the heterogeneous Jobs First effect on welfare participation decision via a partially linear logistic model. To detect treatment effect's heterogeneity, it is essential to use the orthogonal loss rather than the non-orthogonal one.

Literature review. This paper is related to three lines of research: single index models, high-dimensional sparse models, and orthogonal/debiased inference based on machine learning methods. The first line of research concerns with the estimation of single index models (Manski (1975), Powell (1984), Manski (1985), Ichimura (1993), Klein and Spady (1993), Hardle et al. (1993)). This work focuses on a low-dimensional target parameter that can be treated as fixed. Focusing on smooth models, we allow the parameter's dimension to grow with sample size and even exceed it. We show that the single index property is sufficient to ensure the uniform convergence of the sample moments towards its population analog over an $\ell_{1}$-restricted ball, extending the generalization bounds in the machine learning literature (see e.g., Shalev-Shwartz and Ben-David (2014)) to single index CMRs.

The second line of research establishes the finite-sample bounds for a high-dimensional sparse parameter (Belloni et al. (2011), Negahban et al. (2012), Loh and Wainwright (2013), van der Geer et al. (2014), Loh (2017), Zhu (2017), Zhu et al. (2019)). Our contribution is to allow the loss function to depend on a functional nuisance parameter. In the convex case, we establish global convergence of the $\ell_{1}$-regularized $M$-estimator, following Negahban et al. (2012). One could follow a similar path to establish local convergence, building on Loh and Wainwright (2013) and Loh (2017), in the non-convex case. Focusing on the double robustness property, Tan (2020b) and Tan (2020a) establish convergence guarantees in high-dimensional models that are potentially misspecified. After we released the working paper version of this article (arxiv.ID 1702.06240), many methods have proposed similar $M$-estimation approaches. Focusing on missing 
data, Chakrabortty et al. (2019) develops an $M$-estimator, relying on a classical Robins and Rotnitzky (1995) orthogonal score. We derive an orthogonal loss function for an arbitrary single index moment restriction, including Robins and Rotnitzky (1995) as a special case. The follow-up paper by Foster and Syrgkanis (2019) extends our result to $M$-estimators with decomposable regularizers, including $\ell_{1}$ penalty as a special case. An alternative regularized minimum distance approach has been proposed in Belloni et al. (2018).

The third line of research obtains a $\sqrt{N}$-consistent and asymptotically normal estimator of a low-dimensional target parameter $\theta$ in the presence of a nonparametric nuisance parameter (Neyman (1959), Neyman (1979), Hardle and Stoker (1989), Bickel et al. (1993), Newey and Stoker (1993), Andrews (1994),Newey (1994), Robins and Rotnitzky (1995), Ai and Chen (2003)). A statistical procedure is called Neyman-orthogonal if it is locally insensitive with respect to the estimation error of the first-stage nuisance parameter. Combining orthogonality and sample splitting, the Double Machine Learning framework of Chernozhukov et al. (2016) and Chernozhukov et al. (2018) has derived a root$\mathrm{N}$ consistent asymptotically normal estimator of the target parameter based on the firststage machine learning estimates. Extending this work, we establish convergence rates for $\ell_{1}$-regularized $M$-estimators whose loss function gradient is an orthogonal moment. Next, we also contribute to the literature that derives orthogonal moments starting from non-orthogonal ones. Specifically, we construct a bias correction term for a nuisance parameter that is identified by a general conditional exogeneity restriction, covering conditional expectation (Newey (1994)) and conditional quantile (Ichimura and Newey (2015)) as leading special cases. An alternative approach based on automatic debiasing has been proposed in Chernozhukov et al. (2018a) and Chernozhukov et al. (2018b). Finally, we also contribute to the growing literature on orthogonal/doubly robust estimation based on machine learning methods (Sasaki and Ura (2018), Chiang et al. (2019), Sasaki et al. (2020), Chiang (2018)), in particular, heterogeneous treatment effects estimation (Nie and Wager (2017), Semenova et al. (2017), Oprescu et al. (2018), Fan et al. (2019), Zimmert and Lechner (2019), Colangelo and Lee (2020), Semenova and Chernozhukov (2020)). In contrast to this work, our main example features partialling out inside the argument of nonlinear link function, which, to the best of our knowledge, is completely new.

This paper is organized as follows. Section 2 introduces our main examples and gives a non-technical overview of the results. Section 3 formally states our results. Section 4 derives the concrete conditions for our applications. Section 5 gives an empirical application. Appendix A generalizes Theorem 3.1 to the case of extremum estimators beyond $M$-estimators. Appendix B proves Theorem 3.1 and Theorem 3.2. Appendix C verifies the conditions of Theorem 3.1 for each of the three applications.

\section{SET-UP}

We start with the description of a single index conditional moment restriction (CMR) framework. The CMR takes the form

$$
\mathbb{E}\left[\left.m\left(W, \Lambda(Z, \gamma)^{\prime} \theta_{0}, \gamma\right)\right|_{\gamma=g_{0}(Z)} \mid Z=z\right]=0, \quad \forall z,
$$

where the first argument $W \in \mathcal{W} \subseteq \mathrm{R}^{\operatorname{dim} W}$ is the data vector, the second argument $t \in \mathrm{R}$ is the single index, and the third one $\gamma \in \mathrm{R}^{d}$ is the output of the functional nuisance 
parameter. The parameter of interest $\theta \in \mathrm{R}^{p}$ enters the moment function only via its inner product

$$
t=\Lambda(Z, \gamma)^{\prime} \theta,
$$

where the index function $\Lambda(z, \gamma): \mathcal{Z} \times \mathrm{R}^{d} \rightarrow \mathrm{R}^{p}$ is known up to $\gamma$. In many cases (e.g., Ichimura (1993), Klein and Spady (1993)), the index function $\Lambda(z, \gamma)$ reduces to $\Lambda(z, \gamma)=z$. Examples of the nuisance vector-function

$$
g_{0}=g_{0}(z)
$$

include the propensity score, the conditional choice probability, and the regression function, or a combination of these functions. Given the CMR (2.1), our goal is to find a loss function $Q(\theta, \gamma): \mathrm{R}^{p} \times \mathrm{R}^{d} \rightarrow \mathrm{R}$ so that the true parameter value $\theta_{0}$ obeys

$$
\theta_{0}=\arg \min _{\theta} Q\left(\theta, g_{0}\right) .
$$

We find the loss function in two steps. We first solve the ordinary differential equation (ODE)

$$
\frac{\partial}{\partial t} \ell(w, t, \gamma)=m(w, t, \gamma), \quad t \in \mathbb{R} .
$$

We then plug $t=\Lambda(Z, \gamma)^{\prime} \theta$ and $\gamma=g(Z)$ into the sketch of the loss function $\ell(w, t, \gamma)$ to obtain a population loss

$$
Q(\theta, g)=\mathbb{E}\left[\ell\left(W, \Lambda(Z, g(Z))^{\prime} \theta, g(Z)\right)\right],
$$

whose gradient at $g=g_{0}$ and $\theta=\theta_{0}$ is

$$
\nabla_{\theta} Q\left(\theta_{0}, g_{0}\right)=\mathbb{E}\left[\left.m\left(W, \Lambda(Z, \gamma)^{\prime} \theta_{0}, \gamma\right) \cdot \Lambda(Z, \gamma)\right|_{\gamma=g_{0}(Z)}\right] .
$$

In what follows, we refer to $\ell(w, t, \gamma)$ as the loss sketch, to $Q(\theta, g)$ and $\nabla_{\theta} Q(\theta, g)$ as the population loss and the population gradient, to

$$
\widehat{Q}(\theta, \widehat{g}):=\frac{1}{n} \sum_{i=1}^{n} \ell\left(W_{i}, \Lambda\left(Z_{i}, \widehat{g}\left(Z_{i}\right)\right)^{\prime} \theta, \widehat{g}\right)
$$

and to $\nabla_{\theta} \widehat{Q}(\theta, \widehat{g})$ as the sample loss and the sample gradient. The conditional independence assumption (2.1) ensures that (2.5) is a valid moment equation for $\theta_{0}$. If $m(w, t, \gamma)$ is non-decreasing (non-increasing) in $t, \theta_{0}$ is the unique minimizer of $Q\left(\theta, g_{0}\right)\left(-Q\left(\theta, g_{0}\right)\right)$.

The proposed estimator $\hat{\theta}$ has two stages. First, on the auxiliary sample, we construct an estimate $\hat{g}$ of the nuisance parameter $g_{0}$, using a machine learning estimator capable of dealing with the high-dimensional covariate vector $Z$. Second, on the main sample, the target parameter's estimate is taken to be the minimizer of $\ell_{1}$-regularized sample loss. For a fixed vector, its $\ell_{2}$ norm is denoted by $\|\cdot\|_{2}$, the $\ell_{1}$ norm is denoted by $\|\cdot\|_{1}$, the $\ell_{\infty}$ norm is denoted by $\|\cdot\|_{\infty}$, and $\ell_{0}$ norm is denoted by $\|\cdot\|_{0}$.

Definition 2.1 (Regularized $M$-Estimator). Given $\left(\widehat{g}\left(Z_{i}\right)\right)_{i=1}^{n}$ and the penalty parameter $\lambda \geqslant 0$, define

$$
\widehat{\theta}=: \arg \min _{\theta \in \mathrm{R}^{p}} \hat{Q}(\theta, \widehat{g})+\lambda\|\theta\|_{1} .
$$


As discussed in Negahban et al. (2012), the sample gradient $\nabla_{\theta} \widehat{Q}\left(\theta_{0}, \widehat{g}\right)$ at $\theta=\theta_{0}$ summarizes the noise of the problem. If the population gradient (2.5) possesses the orthogonality property (Neyman (1959))

$$
\frac{\partial}{\partial r} \nabla_{\theta} Q\left(\theta_{0}, r\left(g-g_{0}\right)+g_{0}\right)=0, \quad \forall r \in[0,1),
$$

the biased first-stage estimation error $\widehat{g}(Z)-g_{0}(Z)$ has no first-order effect on the sample gradient. As a result, there exists a moderate penalty choice $\lambda=\lambda_{\text {mod }}$ obeying

$$
\lambda_{\text {mod }}=O\left(g_{n}^{2}+\sqrt{\frac{\log p}{n}}\right)
$$

that is sufficiently large to dominate the noise

$$
\lambda / 2 \geqslant\left\|\nabla_{\theta} \widehat{Q}\left(\theta_{0}, \hat{g}\right)\right\|_{\infty} \quad \text { with probability } 1-o(1) .
$$

If (2.8) does not hold, the event (2.10) requires an aggressive choice $\lambda=\lambda_{\text {agg }}$ obeying

$$
\lambda_{\text {agg }}=C\left(g_{n}+\sqrt{\frac{\log p}{n}}\right) .
$$

To sum up, for $C$ sufficiently large, there exists an admissible penalty level $\lambda=\lambda_{\text {adm }}$ obeying (2.10)

$$
\lambda_{\mathrm{adm}}=C\left(\sqrt{\frac{\log p}{n}}+B_{0} g_{n}+g_{n}^{2}\right),
$$

which reduces to $\lambda_{\text {mod }}$ if (2.8) holds (i.e., $B_{0}=0$ ) and to $\lambda_{\text {agg }}$ otherwise. For an admissible penalty choice, Theorem 3.1 establishes the following bounds

$$
\left\|\widehat{\theta}-\theta_{0}\right\|_{2}=O_{P}(\sqrt{k} \lambda), \quad\left\|\hat{\theta}-\theta_{0}\right\|_{1}=O_{P}(k \lambda) .
$$

In particular, if $B_{0}=0$ and $g_{n}^{2}=o(\log p / n)^{1 / 2}$ and $\lambda=\lambda_{\text {adm }}$ obeying (2.12), $\hat{\theta}$ converges at the oracle rate, where the oracle knows the nuisance parameter $g_{0}$ and estimates only $\theta_{0}$. In what follows, if the condition (2.8) holds, we refer to the $m(w, t, \gamma)$ and $\ell(w, t, \gamma)$ as the orthogonal moment and orthogonal loss, respectively.

We conclude this section by studying the partially linear logistic model. The model takes the form

$$
\mathbb{E}\left[Y-G\left(D \cdot\left((1, X)^{\prime} \theta_{0}\right)+f_{0}(X)\right) \mid D, X\right]=0,
$$

where $D \in \mathbb{R}$ is a one-dimensional base treatment, $X \in \mathbb{R}^{p}$ is a vector of controls, $Y$ is a binary outcome, $W=(D, X, Y)$ is the data vector, and $G(t)$ is the logistic link function. The treatment variable $D$ affects the outcome $Y$ via its interactions with the controls $(1, X)$. In addition, $X$ affects $Y$ via the confounding function $f_{0}(X)$ that enters (2.14) in an additively separable way. To make progress, most papers (see e.g., Belloni et al. (2016)) require this function to be linear so that $\theta_{0}$ and the nuisance parameter can be estimated under a joint sparsity assumption. We describe below how to circumvent this bottleneck.

Inspired by Robinson (1988), we propose to partial out the controls inside the link 
function's argument

$$
\mathbb{E}\left[Y-G\left(\left(D-p_{0}(X)\right) \cdot\left((1, X)^{\prime} \theta_{0}\right)+q_{0}(X)\right) \mid D, X\right]=0
$$

where

$$
p_{0}(x)=\mathbb{E}[D \mid X=x]
$$

is the conditional expectation of the treatment and

$$
q_{0}(x)=\mathbb{E}\left[G^{-1}(\mathbb{E}[Y \mid D, X]) \mid X=x\right]
$$

is the conditional expectation of the link function's argument. In contrast to (2.14), the nuisance parameters $p_{0}(x)$ and $q_{0}(x)$ are identified separately from $\theta_{0}$ and permit a wider class of approaches to estimate them. Thus, equation (2.15) is a special case of (2.1) with the conditioning vector $Z=(D, X)$, the moment function

$$
m_{\text {pre }}(w, t, \gamma)=y-G\left(t+\gamma_{2}\right),
$$

the single index $t=\left(d-\gamma_{1}\right) \cdot(1, x)^{\prime} \theta$ and the nuisance parameter $g_{0}(x)=\left\{p_{0}(x), q_{0}(x)\right\}$ whose output is denoted by $\gamma=\left(\gamma_{1}, \gamma_{2}\right)$.

One may be tempted to proceed with the moment function (2.18). Solving the ODE (2.3) gives

$$
\ell_{\text {pre }}(w, t, \gamma)=-y \log G\left(t+\gamma_{2}\right)-(1-y) \log \left(1-G\left(t+\gamma_{2}\right)\right) .
$$

The sample loss $\widehat{Q}(\theta,(\hat{p}, \hat{q}))$ in (2.6) coincides with the negative logistic likelihood. However, the population gradient (2.5) does not obey the orthogonality condition (2.8)

$$
\begin{aligned}
& \frac{\partial}{\partial r} \nabla_{\theta} Q\left(\theta_{0}, r\left(q-q_{0}\right)+q_{0}\right) \\
& =-\mathbb{E} G^{\prime}\left(\left(D-p_{0}(X)\right)\left((1, X)^{\prime} \theta_{0}\right)+q_{0}(X)\right) \cdot\left(D-p_{0}(X)\right)\left(q(X)-q_{0}(X)\right)(1, X)^{\prime} \neq 0 .
\end{aligned}
$$

As a result, the biased estimation error $\widehat{q}(X)-q_{0}(X)$ has a first-order effect on the sample gradient. Thus, the admissible penalty choice reduces to $\lambda_{\mathrm{adm}}=\lambda_{\mathrm{agg}}$ in (2.11), which makes the estimator's rate in (2.13) a slow one.

To restore orthogonality, we reweigh the moment function as in Belloni et al. (2016). The sketch of the new moment function is

$$
m(w, t, \gamma):=\frac{y-G\left(t+\gamma_{2}\right)}{\gamma_{3}}
$$

where $\gamma=\left(\gamma_{1}, \gamma_{2}, \gamma_{3}\right)$ corresponds to the output of $g_{0}(z)=\left\{p_{0}(x), q_{0}(x), V_{0}(d, x)\right\}$ and the weighting function $V_{0}(d, x)$ is the conditional variance

$$
V_{0}(d, x)=G\left(\left(d-p_{0}(x)\right) \cdot\left((1, x)^{\prime} \theta_{0}\right)+q_{0}(x)\right) \cdot\left(1-G\left(\left(d-p_{0}(x)\right) \cdot\left((1, x)^{\prime} \theta_{0}\right)+q_{0}(x)\right)\right)
$$

Solving the ODE (2.3) gives

$$
\ell(w, t, \gamma)=-\frac{1}{\gamma_{3}}\left(y \cdot \log \left(G\left(t+\gamma_{2}\right)\right)+(1-y) \cdot \log \left(1-G\left(\left(t+\gamma_{2}\right)\right)\right)\right) .
$$

The sample loss coincides with the negative weighted logistic likelihood (Belloni et al. 
(2016)). In contrast to (2.20), the population gradient obeys the orthogonality condition (2.8)

$$
\begin{aligned}
& \frac{\partial}{\partial r} \nabla_{\theta} Q\left(\theta_{0}, r\left(q-q_{0}\right)+q_{0}\right) \\
& =-\mathbb{E} \frac{G^{\prime}\left(\left(D-p_{0}(X)\right)\left((1, X)^{\prime} \theta_{0}\right)+q_{0}(X)\right)}{G^{\prime}\left(\left(D-p_{0}(X)\right)\left((1, X)^{\prime} \theta_{0}\right)+q_{0}(X)\right)} \cdot\left(D-p_{0}(X)\right)\left(q(X)-q_{0}(X)\right)(1, X)^{\prime}=0 .
\end{aligned}
$$

As a result, the admissible penalty choice (2.12) reduces to $\lambda_{\mathrm{adm}}=\lambda_{\bmod }$ in (2.9), which makes the estimator's rate in (2.13) a fast one.

\subsection{Examples}

Example 2.1 (Nonlinear Treatment Effects). Suppose the treatment effect $\theta_{0}$ is identified by the conditional moment restriction (2.15), where the link function $G(\cdot): \mathbb{R} \rightarrow \mathbb{R}$ is a known monotone link function that may not necessarily be logistic. Define the weighting function as

$$
V_{0}(d, x)=G^{\prime}\left(\left(d-p_{0}(x)\right) \cdot\left((1, x)^{\prime} \theta_{0}\right)+q_{0}(x)\right) .
$$

The loss sketch $\ell(w, t, \gamma)$ is an arbitrary solution to the ODE

$$
\frac{\partial}{\partial t} \ell(w, t, \gamma)=-\frac{1}{\gamma_{3}}\left(y-G\left(t+\gamma_{2}\right)\right)
$$

where $\Lambda(z, \gamma)=\left(d-\gamma_{1}\right) \cdot(1, x), \gamma=\left(\gamma_{1}, \gamma_{2}, \gamma_{3}\right)$ denotes the output of $g_{0}(z)=\left\{p_{0}(x), q_{0}(x), V_{0}(d, x)\right\}$ defined in (2.16), (2.17) and (2.25). Corollary 4.1 establishes the mean square convergence rate for the Regularized $M$-Estimator based on the loss sketch defined in the ODE (2.26).

Remark 2.1 (Linear Link). Consider Example 2.1 with $G(t)=t$. The function $V_{0}(d, x)$ in (2.25) simplifies to

$$
V_{0}(d, x)=1 .
$$

As a result, the nuisance parameter $g_{0}(z)$ simplifies to $g_{0}(z)=g_{0}(x)=\left\{p_{0}(x), q_{0}(x)\right\}$ and $\gamma=\left(\gamma_{1}, \gamma_{2}\right)$. The moment sketch is

$$
m(w, t, \gamma):=-\left(y-t-\gamma_{2}\right) .
$$

The loss sketch $\ell(w, t, \gamma)$ is

$$
\ell(w, t, \gamma)=\frac{1}{2}\left(y-t-\gamma_{2}\right)^{2},
$$

which corresponds to the least squares loss used in Semenova et al. (2017) and Nie and Wager (2017). The population gradient (2.5) reduces to Robinson (1988)-type score

$$
\nabla_{\theta} Q\left(\theta_{0}, g_{0}\right)=\mathbb{E}\left(Y-\left(D-p_{0}(X)\right) \cdot(1, X)^{\prime} \theta_{0}-q_{0}(X)\right) \cdot\left(D-p_{0}(X)\right) \cdot(1, X)=0 .
$$

Its pathwise derivative with respect to $q$ is zero:

$$
\frac{\partial}{\partial r} \nabla_{\theta} Q\left(\theta_{0}, r\left(q-q_{0}\right)+q_{0}\right)=-\mathbb{E}\left(D-p_{0}(X)\right)\left(q(X)-q_{0}(X)\right) \cdot(1, X)^{\prime}=0 .
$$


Remark 2.2 (Logistic Link). Consider Example 2.1 with $G(t)=\left(1+\exp ^{-t}\right)^{-1}$. The moment sketch is

$$
m(w, t, \gamma):=\frac{y-G\left(t+\gamma_{2}\right)}{\gamma_{3}}
$$

The loss sketch $\ell(w, t, \gamma)$ is

$$
\ell(w, t, \gamma)=-\frac{1}{\gamma_{3}}\left(y \cdot \log \left(G\left(t+\gamma_{2}\right)\right)+(1-y) \cdot \log \left(1-G\left(\left(t+\gamma_{2}\right)\right)\right),\right.
$$

which corresponds to the negative weighted logistic likelihood used in Belloni et al. (2016). The population gradient is

$$
\nabla_{\theta} Q\left(\theta_{0}, g_{0}\right)=-\mathbb{E} \frac{\left(Y-G\left(\left(D-p_{0}(X)\right) \cdot(1, X)^{\prime} \theta_{0}+q_{0}(X)\right)\right.}{V_{0}(D, X)} \cdot\left(D-p_{0}(X)\right) \cdot(1, X),
$$

where $g_{0}(z)=\left(p_{0}(x), q_{0}(x), V_{0}(d, x)\right)$ is as defined in (2.16), (2.17), (2.22).

Example 2.2 (Missing Data). Suppose a researcher is interested in the parameter $\theta_{0}$ identified by a CMR:

$$
\mathbb{E}\left[u\left(Y^{*}, X^{\prime} \theta_{0}\right) \mid X=x\right]=0, \quad \forall x \in \mathcal{X},
$$

where $Y^{*} \in \mathbb{R}$ is a partially observed outcome and $Z=X \in \mathbb{R}^{p}$ is a covariate vector. Let $V \in\{1,0\}$ indicate whether $Y^{*}$ is observed, $Y=V \cdot Y^{*}$ be the observed outcome, and $W=(V, X, Y)$ be the data vector. A standard way to make progress is to assume that $V$ is as good as randomly assigned conditional on $X$.

Define the conditional probability of observing $Y^{*}$ as

$$
p_{0}(x)=\mathbb{E}[V \mid X=x]
$$

and the expectation function $h_{0}(x)$ as

$$
h_{0}(x)=\mathbb{E}\left[u\left(Y, X^{\prime} \theta_{0}\right) \mid X=x, V=1\right] .
$$

The moment sketch is

$$
m(w, t, \gamma)=\frac{v}{\gamma_{1}} u(y, t)-\frac{\gamma_{2}}{\gamma_{1}}\left(v-\gamma_{1}\right),
$$

where $\gamma=\left(\gamma_{1}, \gamma_{2}\right)^{\prime}, \Lambda(x, \gamma)=x$ and $g_{0}(x)=\left\{p_{0}(x), h_{0}(x)\right\}$. The loss sketch is

$$
\ell(w, t, \gamma)=\frac{v}{\gamma_{1}} \ell_{\text {pre }}\left(w, t, \gamma_{1}\right)-\frac{\gamma_{2}}{\gamma_{1}}\left(v-\gamma_{1}\right) t,
$$

where $\ell_{\text {pre }}\left(w, t, \gamma_{1}\right)$ is an arbitrary solution to the ODE

$$
\frac{\partial}{\partial t} \ell_{\text {pre }}\left(w, t, \gamma_{1}\right)=u(y, t) .
$$

The loss (2.32) is a special case of the loss (3.52) proposed in Theorem 3.2. Corollary 4.2 establishes mean square convergence rate for the Regularized $M$-Estimator based on the loss function (2.32)

Remark 2.3 (Quantile Regression with Missing Data). Consider Example 2.2 with

$$
u_{\tau}(y, t)=-\left(1_{\{y \leqslant t\}}-\tau\right),
$$

where $\tau \in(0,1)$ is a quantile level. The moment function (2.34) identifies a quantile 
treatment effect parameter $\theta_{0}$. The loss sketch (2.32) takes the form

$$
\ell(w, t, \gamma):=-\left(\tau \cdot(y-t) 1_{\{y>t\}}+(1-\tau) \cdot(t-y) 1_{\{y<t\}}\right) \frac{v}{\gamma_{1}}-\frac{\gamma_{2}}{\gamma_{1}}\left(v-\gamma_{1}\right) t
$$

Example 2.3 (Static Games of Incomplete Information). Consider a two-player binary choice static game of incomplete information. The utility of action for player one is

$$
U(1)=X^{\prime} \alpha_{0}+V \cdot \Delta_{0}+\epsilon, \quad \mathbb{E}[\epsilon \mid X]=0,
$$

where $X \in \mathbb{R}^{p}$ is the covariate vector, $V \in\{1,0\}$ is the opponent action, $\epsilon$ is mean independent private shock that follows Gumbel distribution. The target $p$-vector $\theta_{0}=\left(\alpha_{0}, \Delta_{0}\right)$ consists of the covariate effect $\alpha_{0}$ and interaction effect $\Delta_{0}$. The utility $U(0)$ of non-action for player one is normalized to zero.

If the players' choices correspond to Bayes-Nash equilibrium, the outcome $Y$ obeys

$$
Y=1\left[X^{\prime} \alpha_{0}+p_{0}(X) \Delta_{0}+\epsilon>0\right],
$$

where $p_{0}(x)=\mathbb{E}[V \mid X=x]$. The moment sketch is

$$
m(w, t, \gamma)=-\left(y-G(t)+\gamma_{2}\left(v-\gamma_{1}\right)\right),
$$

where $\gamma=\left(\gamma_{1}, \gamma_{2}\right)^{\prime}, \Lambda(x, \gamma)=\left(x, \gamma_{1}\right)$, and $g_{0}(x)=\left\{p_{0}(x), h_{0}(x)\right\}$ for $h_{0}(x)=\Delta_{0} G^{\prime}\left(x^{\prime} \alpha_{0}+\right.$ $\left.p_{0}(x) \Delta_{0}\right)$. The loss sketch is

$$
\ell(w, t, \gamma):=\ell_{\text {pre }}\left(w, t, \gamma_{1}\right)-\gamma_{2}\left(v-\gamma_{1}\right) t
$$

where the preliminary loss is the negative logistic likelihood

$$
\ell_{\text {pre }}\left(w, t, \gamma_{1}\right)=-y \cdot G(t)-(1-y) \cdot(1-G(t)) .
$$

The loss (2.37) is a special case of the loss (3.52) proposed in Theorem 3.2. Corollary 4.3 establishes mean square convergence rate for the Regularized $M$-Estimator based on the loss function (2.37).

\subsection{Remarks}

Following the sparsity bounds established in Belloni et al. (2016), we conjecture that the final estimator $\hat{\theta}$ is sparse. Therefore, one can interpret the Lasso estimator $\hat{\theta}$ as a model selector. We expect the post-Lasso-logistic based on single-selection procedure

$$
\widehat{\theta}_{P L}=\arg \min _{\theta \in \mathrm{R}^{p}} \hat{Q}(\theta, \widehat{g}): \quad \operatorname{support}(\theta) \subseteq \operatorname{support}(\hat{\theta})
$$

to be too sensitive to moderate model selection mistakes, occurring when the non-zero coefficients of $\theta_{0}$ are statistically indistinguishable from zero. According to Leeb and Potcher (2005), such inference procedures do not provide a Gaussian approximation that is uniform over the space of $\theta$ and $g_{0}$ and are not honest. Instead, we discuss the following the debiasing procedure of van der Geer et al. (2014).

Remark 2.4 (Debiased Lasso of van der Geer et al. (2014)). Suppose $t \rightarrow m(w, t, \gamma)$ is strictly increasing in $t$ for any $w$ and $\gamma$. Abstracting away from any nuisance components, or, effectively, treating $g_{0}$ as known, the work of van der Geer et al. (2014) proposes a debiased Lasso estimator (eq. 18):

$$
\hat{\theta}_{\text {debiased }}\left(g_{0}\right)=\hat{\theta}-\hat{\Gamma} \frac{1}{n} \sum_{i=1}^{n} m\left(W_{i}, \Lambda\left(Z_{i}, g_{0}\left(Z_{i}\right)\right)^{\prime} \hat{\theta}, g_{0}\right) \cdot \Lambda\left(Z_{i}, g_{0}\left(Z_{i}\right)\right),
$$


where $\widehat{\theta}$ is a preliminary estimator of $\theta_{0}, \Gamma=\left(\nabla_{\theta \theta} Q\left(\theta, g_{0}\right)\right)^{-1}$ is the population Hessian inverse, and $\widehat{\Gamma}$ is the estimator of $\Gamma$. If the matrix $\Gamma$ is sparse, the estimator $\widehat{\Gamma}$ can be constructed by nodewise regression. Unlike the post-selection estimator, $\hat{\theta}_{\text {debiased }}\left(g_{0}\right)$ is Neyman-orthogonal with respect to the bias in the estimation error of $\hat{\theta}-\theta_{0}$ and $\widehat{\Gamma}-\Gamma_{0}$. If the sparsity indices of $\Gamma$ and the parameter $\theta_{0}$ are sufficiently small, van der Geer et al. (2014) shows that the estimator $\widehat{\theta}_{\text {debiased }}\left(g_{0}\right)$ is asymptotically Gaussian. We conjecture that the plug-in estimator

$$
\widehat{\theta}_{\text {debiased }}(\widehat{g})
$$

continues to be asymptotically Gaussian. For the linear link function, the asymptotic normality of $\hat{\theta}_{\text {debiased }}(\widehat{g})$ has been established in Semenova et al. (2017).

\section{THEORETICAL RESULTS}

Notation. We will use the following notation. For two sequences of random variables $a_{n}, b_{n}, n \geqslant 1: a_{n} \lesssim_{P} b_{n}$ means $a_{n}=O_{P}\left(b_{n}\right)$. For two sequences of numbers $a_{n}, b_{n}, n \geqslant 1, a_{n} \lesssim b_{n}$ means $a_{n}=O\left(b_{n}\right)$. Let $T=\left\{j: \quad \theta_{0, j} \neq 0\right\}$ be the set of coordinates of $\theta_{0}$ that are not equal to zero, and let $T^{c}$ be the complement of $T$. For a vector $\delta \in \mathbb{R}^{p}$, let $\left(\delta_{T}\right)_{j}=\delta_{j}$ for each $j \in T$ and $\left(\delta_{T}\right)_{j}=0$ for $j \in T^{c}$. For a vector-valued function $g(z): \mathcal{Z} \rightarrow \mathbb{R}^{d}$, denote its $\ell_{2}$ and $\ell_{\infty}$ norms as

$$
\begin{aligned}
\|g\|_{2} & :=\left(\mathbb{E}\left[\|g(Z)\|_{1}\right]^{2}\right)^{1 / 2}=\left(\mathbb{E}\left(\sum_{l=1}^{d}\left|g_{l}(Z)\right|\right)^{2}\right)^{1 / 2} \\
\|g\|_{\infty} & :=\sup _{z \in \mathcal{Z}}\left\|g(z)-g\left(z^{\prime}\right)\right\|_{1}=\sup _{z \in \mathcal{Z}} \sum_{l=1}^{d}\left|g_{l}(z)-g_{l}\left(z^{\prime}\right)\right| .
\end{aligned}
$$

Furthermore, assume that the index function $\Lambda(z, \gamma): \mathcal{Z} \times \mathbb{R}^{d} \rightarrow \mathbb{R}^{p}$ is sufficiently smooth with respect to $\gamma$, so that the gradient $\nabla_{\gamma} \Lambda_{j}(z, \gamma)$ and the Hessian $\nabla_{\gamma \gamma} \Lambda_{j}(z, \gamma)$ of each coordinate $j \in\{1,2, \ldots, p\}$ are well-defined. Finally, we will use the empirical process notation

$$
\mathbb{E}_{n} f\left(W_{i}\right):=\frac{1}{n} \sum_{i=1}^{n} f\left(W_{i}\right)
$$

Assumption 3.1 (Monotonicity in single index). The moment function $m(w, t, \gamma)$ is nondecreasing in $t$ for any $w \in \mathcal{W}$ and any $\gamma \in \Gamma$.

Assumption 3.1 ensures that the loss sketch $\ell(w, t, \gamma)$ is a convex function of $t$ for any $w$ and $\gamma$. As a result, the sample loss $\theta \rightarrow \widehat{Q}(\theta, \widehat{g})$ defined in (2.7) is a convex function of $\theta$. By convexity, on the event (2.10), the vector of errors $\nu=\hat{\theta}-\theta_{0}$ belongs to the restricted cone

$$
\mathcal{C}(T ; 3)=\left\{\nu \in \mathbb{R}^{p}: \quad\left\|\nu_{T^{c}}\right\|_{1} \leqslant 3\left\|\nu_{T}\right\|_{1}\right\}
$$

as shown in Negahban et al. (2012) (see Lemma S.1). Define the restricted set as

$$
\mathbb{B}=\left\{\theta \in \mathbb{R}^{p}: \theta=\theta_{0}+r \nu, r \in[0,1), \nu \in \mathcal{C}(T ; 3)\right\} .
$$


Define the sample curvature as

$$
\inf _{\theta \in \mathbb{B}, \nu=\theta-\theta_{0}} \frac{\nu^{T} \nabla_{\theta \theta} \hat{Q}(\theta, \hat{g}) \nu}{\|\nu\|_{2}^{2}},
$$

and let the population curvature be the analog of (3.43) based on the population Hessian $\nabla_{\theta \theta} Q\left(\theta, g_{0}\right)$ instead of $\nabla_{\theta \theta} \hat{Q}(\theta, \widehat{g})$.

Assumption 3.2 (Identification). Let $\Sigma$ denote the population covariance matrix of the index function as

$$
\Sigma=\mathbb{E} \Lambda\left(Z, g_{0}(Z)\right) \Lambda\left(Z, g_{0}(Z)\right)^{T} .
$$

Assume that there exists a constant $C_{\min }>0$ so that min eig $\Sigma \geqslant C_{\min }$.

Assumption 3.3 (Bounded derivative on $\mathbb{B}$ ). There exists a constant $B_{\min }>0$ so that the following bound holds:

$$
\inf _{\theta \in \mathbb{B}} \mathbb{E}\left[\left.\nabla_{t} m(W, t, \gamma)\right|_{\gamma=g_{0}(Z), t=\Lambda(Z, \gamma)^{\prime} \theta} \mid Z=z\right] \geqslant B_{\min }>0 \quad \forall z \in \mathcal{Z} .
$$

Assumptions 3.2 with $C_{\min }$ and 3.3 with $B_{\min }$ imply that the population curvature is bounded from below by $\bar{\gamma}=B_{\min } \cdot C_{\min }$ (see Lemma S.6). On the event

$$
\mathcal{V}:=\left\{\sup _{\theta \in \mathbb{B}}\left\|\nabla_{\theta \theta}\left[\hat{Q}(\theta, \hat{g})-Q\left(\theta, g_{0}\right)\right]\right\|_{\infty}<\bar{\gamma} /(32 k)\right\},
$$

the sample curvature (3.43) is bounded from below by $\bar{\gamma} / 2$ (see Lemma S.2). Since

$$
\nabla_{\theta \theta}\left[\widehat{Q}(\theta, \widehat{g})-Q\left(\theta, g_{0}\right)\right] \leqslant\left\|\nabla_{\theta \theta}\left[\widehat{Q}\left(\theta, g_{0}\right)-Q\left(\theta, g_{0}\right)\right]\right\|_{\infty}+\left\|\nabla_{\theta \theta}\left[\widehat{Q}(\theta, \widehat{g})-\widehat{Q}\left(\theta, g_{0}\right)\right]\right\|_{\infty},
$$

it suffices to show that each summand above is $o_{P}(1)$. We bound the first and the second summand in Lemmas 3.1 and S.8, respectively.

Assumption 3.4 ensures that the moment function is sufficiently smooth in its second and third arguments. Let $\mathcal{W}$ be an open bounded set containing the support of the data vector $W$. Likewise, let $\mathcal{T}$ be an open set containing the support of $\Lambda\left(Z, g_{0}(Z)\right)^{\prime} \theta_{0}$. Finally, let $\Gamma$ be an open bounded set containing the support of vector $g(Z)$, when $g \in \mathcal{G}_{n}$. In what follows, we assume that the sets $\mathcal{W}, \mathcal{T}, \Gamma$ do not change with $n$.

Assumption 3.4 (Smooth and bounded design). There exists a constant $U<\infty$ so that $\sup _{\theta \in \Theta}\|\theta\|_{1} \leqslant U$ and for any vector $w \in \mathcal{W}$, number $t \in \mathcal{T}$ and vector $\gamma \in \Gamma$ the following conditions hold:

$$
\begin{aligned}
|m(w, t, \gamma)|,\left\|\nabla_{\gamma} m(w, t, \gamma)\right\|_{\infty},\left|\nabla_{t} m(w, t, \gamma)\right| & \leqslant U, \\
\left\|\nabla_{\gamma t} m(w, t, \gamma)\right\|_{\infty},\left\|\nabla_{\gamma \gamma} m(w, t, \gamma)\right\|_{\infty},\left|\nabla_{t t} m(w, t, \gamma)\right| & \leqslant U, \\
\left\|\Lambda_{j}(z, \gamma)\right\|_{\infty},\left\|\nabla_{\gamma} \Lambda_{j}(z, \gamma)\right\|_{\infty},\left\|\nabla_{\gamma \gamma} \Lambda_{j}(z, \gamma)\right\|_{\infty} & \leqslant U, \quad \forall j \in\{1,2, \ldots, p\} .
\end{aligned}
$$

Lemma 3.1 establishes a convergence rate for the sample Hessian uniformly over the restricted set (3.41). A gradient version of Lemma 3.1 is available in the concurrent work by Belloni et al. (2018).

Lemma 3.1 (Uniform Convergence of Sample Hessian). Suppose Assumption 3.4 holds 
with a constant $U$. Then, there exists a sequence $\tau_{n}=4 k U^{5} \sqrt{\frac{2 \log p}{n}}+2 U^{3} \sqrt{\frac{2 \log p}{n}}=o(1)$ so that $\nabla_{\theta \theta} \hat{Q}\left(\theta, g_{0}\right)$ uniformly converges to $\nabla_{\theta \theta} Q\left(\theta, g_{0}\right)$ :

$$
\sup _{\theta \in \mathrm{B}}\left\|\nabla_{\theta \theta} \widehat{Q}\left(\theta, g_{0}\right)-\nabla_{\theta \theta} Q\left(\theta, g_{0}\right)\right\|_{\infty}=O_{P}\left(\tau_{n}\right)=o_{P}(1) .
$$

Proof. Consider the function class

$$
\mathcal{F}=\left\{Z \rightarrow \Lambda\left(Z, g_{0}(Z)\right)^{\prime} \theta, \quad \theta \in \mathbb{R}^{p}, \quad\|\theta\|_{1} \leqslant k U\right\} .
$$

Define its Rademacher complexity

$$
\mathcal{R}(\mathcal{F}):=\mathbb{E}\left[\sup _{\theta:\|\theta\|_{1} \leqslant k U} \mathbb{E}_{n} \sigma_{i} \Lambda\left(Z_{i}, g_{0}\left(Z_{i}\right)\right)^{\prime} \theta\right],
$$

where $\left(\sigma_{i}\right)_{i=1}^{n}$ is a vector of i.i.d random variables from Rademacher distribution: $\mathrm{P}\left(\sigma_{i}=\right.$ $+1)=\mathrm{P}\left(\sigma_{i}=-1\right)=0.5$. Each function in the class

$$
\mathcal{H}=\left\{W \rightarrow \nabla_{\theta_{k} \theta_{j}} \ell\left(W, \Lambda\left(Z, g_{0}(Z)\right)^{\prime} \theta, g_{0}(Z)\right), \quad \theta \in \mathbb{R}^{p},\|\theta\|_{1} \leqslant k U\right\}
$$

is a combination of the function in the class $\mathcal{F}$ and a $U^{3}$-Lipshitz function. Invoking Contraction Lemma (Lemma 26.9) and Lemma 26.11 from Shalev-Shwartz and Ben-David (2014), we obtain

$$
\mathcal{R}(\mathcal{H}) \leqslant U^{3} \mathcal{R}(\mathcal{F}) \leqslant U^{5} \cdot k \sqrt{\frac{2 \log (2 p)}{n}} .
$$

Finally, invoking Lemma 26.5 Shalev-Shwartz and Ben-David (2014) for each pair $(k, j) \in$ $\{1, \ldots, p\}^{2}$, w.p. $1-\delta / p^{2}$,

$$
\begin{aligned}
& \sup _{\|\theta\|_{1} \leqslant k U}\left|\left[\mathbb{E}_{n}-\mathbb{E}\right]\left[\nabla_{\theta_{k} \theta_{j}} \ell\left(W_{i}, \Lambda\left(Z_{i}, g_{0}\left(Z_{i}\right)\right)^{\prime} \theta_{0}, g_{0}\left(Z_{i}\right)\right)\right]\right| \\
& \leqslant 2 \mathcal{R}(\mathcal{H})+U^{3} \sqrt{\frac{2 \log \left(2 p^{3} / \delta\right)}{n}} \\
& \leqslant 2 k U^{5} \sqrt{\frac{2 \log \left(2 p^{3} / \delta\right)}{n}}+U^{3} \sqrt{\frac{2 \log \left(2 p^{3} / \delta\right)}{n}} .
\end{aligned}
$$

Union bound over $p^{2}$ pairs $(k, j) \in\{1, \ldots, p\}^{2}$ and $2 p^{3} \leqslant p^{4}$ gives w.p. $1-\delta$

$$
\begin{aligned}
& \sup _{1 \leqslant k, j \leqslant p} \sup _{\|\theta\|_{1} \leqslant k U}\left|\left[\mathbb{E}_{n}-\mathbb{E}\right]\left[\nabla_{\theta_{k} \theta_{j}} \ell\left(W_{i}, \Lambda\left(Z_{i}, g_{0}\left(Z_{i}\right)\right)^{\prime} \theta_{0}, g_{0}\left(Z_{i}\right)\right)\right]\right| \\
& \leqslant 4 k U^{5} \sqrt{\frac{2 \log (p / \delta)}{n}}+2 U^{3} \sqrt{\frac{2 \log (p / \delta)}{n}}
\end{aligned}
$$

Assumption 3.5 formalizes the convergence of the nuisance parameter's estimator. It introduces a sequence of nuisance realization sets $\mathcal{G}_{n} \subseteq \mathcal{G}$ that contain the true value $g_{0}$ and the estimator $\hat{g}$ with probability $1-\delta_{n}$. As the sample size $n$ increases, the sets $\mathcal{G}_{n}$ shrink. The shrinkage speed is measured by the rate $g_{n}$ and is referred to as the first-stage rate.

Assumption 3.5 (First-stage rate). There exist sequences of numbers $\delta_{n}=o(1)$ and $g_{n}=o(1)$ 
and a sequence of sets $\mathcal{G}_{n} \subseteq \mathcal{G}$ so that $\hat{g} \in \mathcal{G}_{n}$ w.p. at least $1-\delta_{n}$ and $g_{0} \in \mathcal{G}_{n}$. The sets shrink at the rate

$$
\sup _{g \in \mathcal{G}_{n}}\left\|g-g_{0}\right\| \leqslant g_{n},
$$

where $\|\cdot\|$ is either the $\ell_{\infty}$ norm or the $\ell_{2}$ norm as defined in equation (3.40).

Assumption 3.5 is satisfied by many machine learning estimators under structural assumptions on the model in $\ell_{2}$ and/or $\ell_{\infty}$ norm. For example, it holds for Lasso (Belloni et al. (2011), Belloni and Chernozhukov (2013)) in linear and generalized linear models, for $L_{2}$-boosting in sparse models (Luo and Spindler (2016)), for neural network (Chen and White (1999)), and for random forest in low-dimensional (Wager and Walther (2015)) and high-dimensional sparse (Syrganis and Zampetakis (2020)) models. For a broad overview of low-level primitive conditions that covers neural networks and random forest see, e.g., Jeong and Namkoong (2020), Appendix 1.

Theorem 3.1 (Regularized $M$-Estimator). Suppose that the nuisance space $\mathcal{G}$ is equipped with either the $\ell_{\infty}$ or the $\ell_{2}$ norm, defined in equation (3.40). Suppose Assumptions 3.1-3.5 hold, and $k\left(g_{n}+U^{5} \cdot k \cdot \sqrt{\frac{2 \log 2 p}{n}}+3 d \cdot U^{3} \sqrt{\frac{\log (2 p)}{n}}\right)=o(1)$. Then, for $n$ and $C$ large enough and the admissible penalty $\lambda=\lambda_{a d m}$ is chosen to obey (2.12), Regularized M-Estimator obeys the bound (2.13).

Theorem 3.1 is our main result. It establishes the convergence rate for the Regularized $M$-estimator, covering the orthogonal (i.e., $B_{0}=0$ ) and the non-orthogonal (i.e., $B_{0} \neq$ $0)$ cases. In the former case, the admissible penalty choice $\lambda_{\text {adm }}$ reduces to $\lambda_{\bmod }$ in (2.9), which makes the estimator's rate (2.13) fast. Otherwise, $\lambda_{\text {adm }}$ reduces to $\lambda_{\text {agg }}$ in (2.11), which makes the estimator's rate (2.13) slow. In all our examples, we invoke Theorem 3.1 twice: first, for the preliminary estimate $\check{\theta}$ based on a non-orthogonal CMR (3.47) and, second, for the final estimate $\hat{\theta}$ based on the orthogonal CMR.

Suppose that the moment function $m_{\text {pre }}\left(w, t, \gamma_{1}\right): \mathcal{W} \times \mathbb{R} \times \mathbb{R} \rightarrow \mathbb{R}$ and/or the index function $\Lambda\left(z, \gamma_{1}\right): \mathcal{W} \times \mathbb{R} \rightarrow \mathbb{R}^{p}$ depend on a one-dimensional functional nuisance parameter $p_{0}(z)$ :

$$
\mathbb{E}\left[m_{\text {pre }}\left(W, \Lambda\left(Z, p_{0}(Z)\right)^{\prime} \theta_{0}, p_{0}(Z)\right) \mid Z=z\right]=0, \quad z \in \mathcal{Z} .
$$

Furthermore, suppose the nuisance parameter is identified by a conditional exogeneity restriction

$$
\mathbb{E}\left[R\left(W, p_{0}(Z)\right) \mid Z=z\right]=0 .
$$

For example, $R(W, p(X))=D-p(X)$ defines the conditional expectation function as in (2.16), and $R(W, p(X))=1_{\{V \leqslant p(X)\}}-\tau$ defines the conditional $\tau$-quantile function. Starting from an arbitrary CMR (3.47), Theorem 3.2 derives a loss whose gradient obeys the orthogonality condition (2.8).

Theorem 3.2 (Construction of Orthogonal Loss). Suppose equation (3.48) holds. Define the adjusted moment function

$$
m(w, t, \gamma)=m_{\text {pre }}\left(w, t, \gamma_{1}\right)-\gamma_{2} \cdot \gamma_{3}^{-1} \cdot R\left(w, \gamma_{1}\right)
$$


where $\gamma=\left(\gamma_{1}, \gamma_{2}, \gamma_{3}\right)$ denotes the output of the nuisance parameter $g_{0}(z)=\left\{p_{0}(z), h_{0}(z), I_{0}(z)\right\}$, consisting of $p_{0}(z)$ as defined in $(3.48), h_{0}(z)$ and $I_{0}(z)$ defined as

$$
\begin{aligned}
& h_{0}(z)=\mathbb{E}\left[\left.\nabla_{\gamma_{1}} m\left(W, t, \gamma_{1}\right)\right|_{\gamma_{1}=p_{0}(Z), t=\Lambda\left(Z, \gamma_{1}\right)^{\prime} \theta_{0}} \mid Z=z\right] \\
& I_{0}(z)=\mathbb{E}\left[\left.\nabla_{\gamma_{1}} R\left(W, \gamma_{1}\right)\right|_{\gamma_{1}=p_{0}(Z)} \mid Z=z\right] .
\end{aligned}
$$

The loss sketch $\ell(w, t, \gamma)$ takes the form

$$
\ell(w, t, \gamma)=\ell_{\text {pre }}\left(w, t, \gamma_{1}\right)-\gamma_{2} \cdot \gamma_{3}^{-1} \cdot R\left(w, \gamma_{1}\right) \cdot t,
$$

where $\ell_{\text {pre }}\left(w, t, \gamma_{1}\right)$ solves the Ordinary Differential Equation (2.3) for the moment function $m_{\text {pre }}\left(w, t, \gamma_{1}\right)$. Then, the population loss $Q\left(\theta, g_{0}\right)$ defined in (2.2) obeys the orthogonality condition (2.8).

$$
\begin{gathered}
\text { Suppose } \sup _{w \in \mathcal{W}} \sup _{\gamma_{1} \in \Gamma_{1}}\left|R\left(w, \gamma_{1}\right)\right| \leqslant U, \sup _{w \in \mathcal{W}} \sup _{\gamma_{1} \in \Gamma_{1}}\left|\nabla_{\gamma_{1}} R\left(w, \gamma_{1}\right)\right| \leqslant U, \\
\qquad \sup _{w \in \mathcal{W}} \sup _{\gamma_{1} \in \Gamma_{1}}\left|\nabla_{\gamma_{1} \gamma_{1}} R\left(w, \gamma_{1}\right)\right| \leqslant U .
\end{gathered}
$$

If the original moment function $m_{\text {pre }}\left(w, t, \gamma_{1}\right)$ obeys Assumptions 3.1-3.4, the adjusted moment function (3.49) obeys Assumptions 3.1-3.4.

Starting from an arbitrary CMR (3.47), Theorem 3.2 adjusts the moment function so that population gradient $\nabla_{\theta} Q\left(\theta_{0}, g_{0}\right)$ obeys orthogonality condition (2.8). Special cases of equation (3.48), such as the conditional expectation function and conditional quantile function, are available in Newey (1994), Chernozhukov et al. (2016) and Ichimura and Newey (2015) for unconditional moment problems. We extend the results above to allow for an arbitrary conditional exogeneity restriction.

\section{APPLICATIONS}

In all the applications below, our starting point is the CMR (3.47) which does not obey the orthogonality condition (2.8). Invoking either the weighting idea (Example 2.1) or Theorem 3.2 (Examples 2.2-2.3), we derive an orthogonal CMR obeying (2.8). However, this CMR requires an additional preliminary estimate of $\theta_{0}$ on top of the nuisance parameters involved in (3.47). Definition 4.1 describes the suggested two-step procedure, spelling out the choices of main/auxiliary samples and the penalty parameters in each step.

Definition 4.1 (Two-Step Regularized $M$-estimator). Let $K=3$ denote the 3 -fold partition of the sample $\{1,2, \ldots, n\}$ into $J_{1}, J_{2}, J_{3}$. For notational convenience, let $J_{0}=J_{3}$ and $J_{4}=J_{1}$. For each $k \in\{0,1,2\}$, compute

1 With $J_{k-1}$ as the auxiliary sample and $J_{k}$ as the main sample, let $\check{\theta}$ be the output of Definition 2.1 with the preliminary loss $\ell_{\text {pre }}\left(w, t, \gamma_{1}\right)$ and the penalty parameter $\lambda_{\text {agg }}$ as in (2.11). For each $i \in J_{k+1}$, the nuisance parameter $\widehat{g}\left(Z_{i}\right)$ is evaluated at $\widehat{g}\left(Z_{i}\right)=\widehat{g}_{k}\left(Z_{i}\right)=\left\{\widehat{p}_{k-1}\left(Z_{i}\right), \check{\theta}_{k}\right\}$.

2 With $\left(J_{k-1}, J_{k}\right)$ as the auxiliary sample and $J_{k+1}$ as the main sample, let $\hat{\theta}$ be the output of Definition 2.1 with the final loss $\ell(w, t, \gamma)$ and the admissible penalty parameter $\lambda_{\text {mod }}$ obeying (2.9). Report: $\hat{\theta}$ 


\subsection{Nonlinear Treatment Effects}

Consider Example 2.1. Define the covariance matrix

$$
\Sigma_{\mathrm{TE}}:=\mathbb{E}\left[\left(D-p_{0}(X)\right)\left(D-p_{0}(X)\right)(1, X)(1, X)^{T}\right] .
$$

Assumption 4.6 (Regularity Conditions for Nonlinear Treatment Effects). Suppose the following conditions hold.

1 (Identification) There exists a constant $\gamma_{T E}>0$ so that min eig $\Sigma_{T E} \geqslant \gamma_{T E}$.

2 (Smooth and Bounded Design). The parameter space $\Theta$ is bounded in $\ell_{1}$ norm by a constant $H_{T E} \geqslant 1: \sup _{\theta \in \Theta}\|\theta\|_{1} \leqslant H_{T E}$ and $\sup _{w \in \mathcal{W}}\|w\|_{\infty} \leqslant H_{T E}$. The functions $t \rightarrow$ $G(t), G^{\prime}(t), G^{(2)}(t), G^{3}(t)$ are $U_{T E}$-bounded on the set $\left[-3 \cdot H_{T E}^{3}, 3 \cdot H_{T E}^{3}\right]$, where $U_{T E} \geqslant$ $H_{T E}$. Furthermore, the functions $t \rightarrow G^{-1}(t),\left(G^{\prime}\right)^{-1}(t),\left(G^{\prime}\right)^{-2}(t),\left(G^{\prime}\right)^{-3}(t)$ are $U_{T E^{-}}$ bounded from above on the set $\left[-3 \cdot H_{T E}^{3}, 3 \cdot H_{T E}^{3}\right]$. Finally, $t \rightarrow G^{\prime}(t)$ is $B_{\text {min }}$-bounded from below on the set $\left[-3 \cdot H_{T E}^{3}, 3 \cdot H_{T E}^{3}\right]$.

3 (First-Stage Rate). There exists an estimator $(\hat{p}(x), \widehat{q}(x))$ of $\left(p_{0}(x), q_{0}(x)\right)$ satisfying Assumption 3.5 with $\pi_{n}+q_{n}$ rates in either $\ell_{\infty}$ or $\ell_{2}$ norm.

4 (Monotonicity). The function $G(t): \mathbb{R} \rightarrow \mathbb{R}$ is a known monotone function of $t$.

Remark 4.5 (Weighting function estimator). For $k \in\{0,1,2\}$ and $i \in J_{k+1}$, define the estimator $\widehat{V}\left(D_{i}, Z_{i}\right)=\widehat{V}_{k}\left(D_{i}, Z_{i}\right)$

$$
\widehat{V}_{k}\left(D_{i}, X_{i}\right)=G^{\prime}\left(\left(D_{i}-\widehat{p}_{k}\left(X_{i}\right)\right) \cdot\left(1, X_{i}\right)^{\prime} \check{\theta}_{k}+\widehat{q}_{k}\left(X_{i}\right)\right), \quad i \in J_{k+1} .
$$

where preliminary loss $\ell_{\text {pre }}\left(w, t, \gamma_{1}\right)$ as in (2.19) is used for $\check{\theta}$.

Corollary 4.1 (Nonlinear Treatment Effects). Suppose Assumption 4.6 holds. Let the preliminary loss $\ell_{\text {pre }}\left(w, t, \gamma_{1}\right)$ be as in (2.19) and the first-stage parameter estimate $(\hat{\pi}(x), \hat{q}(x))$. Let the final loss be as in (2.26) and $\hat{g}(d, x)=(\hat{\pi}(x), \hat{q}(x), \hat{V}(d, x))$, where $\hat{V}(d, x)$ as defined in (4.54).Then, the statement of Theorem 3.1 holds for each step of Definition 4.1, where the first-step nuisance rate is $O\left(\pi_{n}+q_{n}\right)$ and the second-step nuisance rate is $g_{n}=O\left(k\left(\pi_{n}+q_{n}+\sqrt{\frac{\log p}{n}}\right)\right)$.

\subsection{Missing Data}

Consider Example 2.2. Define the covariance matrix

$$
\Sigma_{M D}=\mathbb{E} X X^{T}
$$

and the function $q(t, x)$ as

$$
q_{0}(x, t)=\mathbb{E}\left[\nabla_{t} u(Y, t) \mid V=1, X=x\right] .
$$

Assumption 4.7 (Regularity Conditions for Missing Data). Suppose the following conditions hold.

1 (Identification). There exists $\gamma_{M D}>0$ such that min eig $\Sigma_{M D} \geqslant \gamma_{M D}$. Furthermore, there exists $B_{\text {min }}>0$ so that $\inf _{t \in \mathcal{T}} \mathbb{E}\left[\nabla_{t} u(Y, t) \mid X=x\right] \geqslant B_{\text {min }}$ for any $x \in \mathcal{X}$.

2 (Overlap Condition). There exists a constant $\bar{p}>0$ such that $\inf _{x \in \mathcal{X}} p_{0}(x) \geqslant p>0$. 
3 (Smooth and Bounded Design). The parameter space $\Theta$ is bounded in $\ell_{1}$ norm by a constant $H_{M D}: \sup _{\theta \in \Theta}\|\theta\|_{\infty} \leqslant H_{M D}$ and $\sup _{w \in \mathcal{W}}\|w\|_{\infty} \leqslant H_{M D}$. The functions $u(y, t), \nabla_{t} u(y, t)$, $\nabla_{t t} u(y, t)$ are $U_{M D}$-bounded in $\ell_{\infty}$ norm for all $w \in \mathcal{W}$ and $t \in \mathcal{T}$, where $U_{M D} \geqslant H_{M D}$.

4 (First-Stage Rate). There exists an estimator $(\hat{p}(x), \hat{q}(x, t))$ of $\left(p_{0}(x), q_{0}(x, t)\right)$ satisfying Assumption 3.5 with $\pi_{n, r}+q_{n, r}$ rates in either $\ell_{\infty}$ or $\ell_{2}$ norm, where $q_{n, r}$ rate is

$$
q_{n, \infty}:=\sup _{q \in \mathcal{Q}_{n}} \sup _{t \in R} \sup _{x \in \mathcal{X}}\left|q(x, t)-q_{0}(x, t)\right|, \quad q_{n, 2}:=\sup _{q \in \mathcal{Q}_{n}} \sup _{t \in R}\left(\mathbb{E}\left(q(X, t)-q_{0}(X, t)\right)^{2}\right)^{1 / 2} .
$$

5 (Monotonicity). The function $u(y, t)$ is increasing in $t$ for any $y \in \mathbb{R}$.

Remark 4.6 (First-Stage Estimator of $h_{0}(x)$ ). For each $i \in J_{k+1}$, define the estimator $\widehat{h}\left(X_{i}\right)=\widehat{h}_{k}\left(X_{i}\right)$

$$
\widehat{h}\left(X_{i}\right)=\widehat{q}_{k}\left(X_{i}^{\prime} \check{\theta}_{k}, X_{i}\right)
$$

where the preliminary estimator $\check{\theta}$ is based on the loss $\ell_{\text {pre }}\left(w, t, \gamma_{1}\right)$ as in (2.33).

Corollary 4.2 (General Moment Problems with Missing Data). Suppose Assumption 4.7 holds. Let the preliminary loss $\ell_{\text {pre }}\left(w, t, \gamma_{1}\right)$ be as in (2.33) and the first-stage parameter estimate $\hat{p}(x)$. Let the final loss be as in (2.32) and $\widehat{g}(x)=(\widehat{p}(x), \widehat{h}(x))$, where $\widehat{h}(x)$ as defined in (4.56). Then, the statement of Theorem 3.1 holds for each step of Definition 4.1, where the first-step nuisance rate is $O\left(\pi_{n}\right)$ and the second-step nuisance rate $g_{n}=O\left(k\left(\pi_{n}+\sqrt{\frac{\log p}{n}}\right)+q_{n}\right)$.

\subsection{Static Games of Incomplete Information}

Consider Example 2.3. Define the covariance matrix

$$
\Sigma_{\text {games }}=\mathbb{E}\left[\left(X, p_{0}(X)\right)\left(X, p_{0}(X)\right)^{T}\right] .
$$

Assumption 4.8 (Regularity Conditions for Static Games of Incomplete Information). Suppose the following conditions hold.

1 (Identification). There exists $\gamma_{g a m e s}>0$ so that min eig $\Sigma_{\text {games }} \geqslant \gamma_{\text {games }}$.

2 (Smooth and Bounded Design). There exists a constant $H_{\text {games }}<\infty$ so that $\sup _{\theta \in \Theta}\|\theta\|_{\infty} \leqslant$ $H_{\text {games }}$ and $\sup _{x \in \mathcal{X}}\|x\|_{\infty} \leqslant H_{\text {games. }}$. In addition, $\sup _{\theta \in \Theta}\|\theta\|_{1} \leqslant H_{\text {games }}$.

3 There exists an estimator $\hat{p}(x)$ obeying Assumption 3.5 with $\pi_{n}$ rates in either $\ell_{\infty}$ or $\ell_{2}$ norm.

Remark 4.7 (First-Stage Estimator of $h_{0}(x)$ ). For each $i \in J_{k+1}$, define the estimator $\widehat{h}\left(X_{i}\right)=\widehat{h}_{k}\left(X_{i}\right)$

$$
\widehat{h}\left(X_{i}\right)=\widetilde{\Delta}_{k} \cdot G_{k}^{\prime}\left(X_{i}^{\prime} \check{\alpha}_{k}+\check{\Delta}_{k} \cdot \widehat{p}\left(X_{i}\right)\right)
$$

where the preliminary estimator $\check{\theta}=(\check{\alpha}, \check{\Delta})$ is based on the loss $\ell_{\text {pre }}\left(w, t, \gamma_{1}\right)$ as in (2.38).

Corollary 4.3 (Games of Incomplete Information). Suppose Assumption 4.7 holds. Let the preliminary loss $\ell_{\text {pre }}\left(w, t, \gamma_{1}\right)$ be as in (2.38) and the first-stage parameter estimate $\hat{p}(x)$. Let the final loss be as in (2.37) and $\widehat{g}(x)=(\widehat{p}(x), \hat{h}(x))$, where $\widehat{h}(x)$ as defined in (4.58). Then, the 
statement of Theorem 3.1 holds for each step of Definition 4.1, where the first-step nuisance rate is $O\left(\pi_{n}\right)$ and the second-step nuisance rate $g_{n}=O\left(k\left(\pi_{n}+\sqrt{\frac{\log p}{n}}\right)\right)$.

\section{EMPIRICAL APPLICATION}

In this section, we study the short-term impact of Connecticut's Jobs First welfare reform experiment on women's labor supply and welfare participation decisions. Jobs First, a welfare-to-work assistance program, was introduced in Connecticut in the late 1990s as an alternative to the federal Aid to Families with Dependent Children (AFDC) program. Imposing revealed preference restrictions, Kline and Tartari (2016)'s nonparametric bounds show that Jobs First induced many women to work but led some others to reduce their earnings in order to receive assistance. To gain more insight into the question, we postulate a partially linear logistic model for women's welfare participation decision and estimate heterogeneous Jobs First effects.

The data for our analysis are the same as in Kline and Tartari (2016). They come from Manpower Demonstration Research Corporation (MDRC). In 1996, MDRC conducted a randomized trial that randomly selected a set of eligible female applicants into Jobs First (the treatment group), leaving the remaining females eligible for AFDC (the control group). The outcome of interest is the binary indicator that is equal to one if a woman receives any type of welfare (e.g., AFDC or food stamps) at the fourth quarter after random assignment. Baseline characteristics include constant, age, education level, quarterly history of employment, earnings, AFDC, and food stamps during 8 quarters before random assignment (RA). We postulate a logistic specification with a partially linear index

$$
\mathrm{P}(Y=1 \mid D=d, X=x)=G\left(d \cdot\left((1, x)^{\prime} \theta_{0}\right)+f_{0}(x)\right),
$$

where $D=1$ if a woman is assigned to Jobs First and $Y=1$ if a woman is on welfare. We assume that the treatment $D$ and the controls $X$ affect the outcome $Y$ via an additively separable index

$$
D \cdot\left((1, X)^{\prime} \theta_{0}\right)+f_{0}(X)
$$

entering the logistic link function $G(\cdot): \mathbb{R} \rightarrow \mathbb{R}$. The base treatment $D$ enters the index (5.60) through its interactions with the control vector $(1, X)$. In addition, the control vector $X$ enters the index (5.60) through an unknown function $f_{0}(x)$, which summarizes the confounding effect of the controls on $Y$. Our main object of interest is the heterogeneous treatment effects vector

$$
\theta_{0}=\left(\theta_{0,1}, \theta_{0,-1}\right)^{\prime},
$$

where $\theta_{0,1}$ is the baseline treatment effect and $\theta_{0,-1}$ is the treatment interaction effect. We assume that, out of 20 treatment effect interactions, only few have non-zero value, but do not know their identities.

A standard approach to this problem is to require the confounding function $f_{0}(x)$ to be a linear sparse function of the controls

$$
f_{0}(x)=B(x)^{\prime} \alpha_{0}, \quad p_{\alpha}=\operatorname{dim}\left(\alpha_{0}\right) \gg n, \quad\left\|\alpha_{0}\right\|_{0}=k_{\alpha} \ll n,
$$

where $B(x)$ is a vector of basis functions of $x$ and $n$ is the sample size. We take $B(x)$ to be a vector of $p_{\alpha}=1,600$ pairwise interactions of the controls. The joint estimate of $\theta_{0}$ 
and $\alpha_{0}$ is

$$
\begin{aligned}
& \left(\check{\theta}_{\text {direct }}, \widetilde{\alpha}_{\text {direct }}\right)= \\
& \arg \min _{(\theta, \alpha) \in \mathbb{R}^{p+p_{\alpha}}} \frac{1}{n} \sum_{i=1}^{n}-Y_{i}\left(D_{i} \cdot\left(\left(1, X_{i}\right)^{\prime} \theta\right)+B\left(X_{i}\right)^{\prime} \alpha\right)+\log \left(1+\exp \left(D_{i} \cdot\left(\left(1, X_{i}\right)^{\prime} \theta\right)+B\left(X_{i}\right)^{\prime} \alpha\right)\right) \\
& +\lambda_{\text {direct }}\left\|\theta_{-1}\right\|_{1}+\lambda_{\text {direct }}\|\alpha\|_{1},
\end{aligned}
$$

where the penalty parameter $\lambda_{\text {direct }}$ recommended by Belloni et al. (2016) is

$$
\lambda_{\text {direct }}=\frac{1.1}{2 \sqrt{n}} \Phi^{-1}\left(1-0.05 /\left(p+p_{\alpha}\right) \log (n)\right)=0.036
$$

Following Tan (2020a), we do not penalize the first coordinate $\theta$, imposing the sparsity assumption on the heterogeneous modification effects but not the level of the treatment effect for the baseline category.

We compare the direct estimator to the Regularized $M$-Estimator $\hat{\theta}$, described in Section 4.1. This estimator no longer requires the control function $f_{0}(x)$ to be sparse with respect to the chosen basis $B(x)$. Instead, we assume that the conditional probability of welfare

$$
G_{0}(d, x)=\mathbb{E}[Y \mid D=d, X=x]
$$

can be well-approximated by trees. While this assumption is less interpretable, it allows $G_{0}(d, x)$ to have a nonlinear argument. We estimate $G_{0}(d, x)$ by the probability random forest as in Malley et al. (2012) with 100 trees and default size of leaf node. As for $q_{0}(x)$, we take

$$
\widehat{q}(x)=\widehat{\mathbb{E}}\left[\log \frac{\widehat{G}(d, x)}{1-\widehat{G}(d, x)} \mid X=x\right],
$$

where the outer expectation function is estimated by regular random forest of Breiman (2001). In addition, we assume that the propensity score $p_{0}(x)$ in equation (2.16) is a sufficiently smooth function of $x$ and estimate it by simple logistic regression. The weighting function $\widehat{V}(d, x)$ is estimated as in (4.54). In Definition 2.1, we set $\lambda$ to be

$$
\lambda=\lambda_{\text {ortho }}=\frac{1.1}{2 \sqrt{n}} \Phi^{-1}(1-0.05 / n)=0.031
$$

standardizing each covariate after interacting it with treatment. The Lasso estimate $\hat{\theta}$ and its post-penalized analog post-Lasso-logistic $\hat{\theta}_{\mathrm{PL}}$ are reported in Table 1, Columns (2)-(3). Finally, we report the unpenalized version of the orthogonal estimator defined as

$$
\widehat{\theta}_{\text {unpenalized }}=\arg \min _{\theta \in \mathrm{R}^{p}} \widehat{Q}(\theta, \widehat{g})
$$

Since the number of treatment interactions $p=20$ is less than the sample size, this estimator is well-defined. We report this estimate and the standard errors in Table 1, Columns (4)-(5). Our method is straightforward to implement using the glmnet function in the glmnet $R$ package, setting the off set argument to $\widehat{q}(x)$, the weights argu- 
ment to $\widehat{V}(d, x)$, and the penalty. factor $=(0,1,1, \ldots, 1)$ to accommodate the penalty in $(5.62)^{1}$.

Our empirical findings are as follows. The direct estimator (Column (1)) implies that Jobs First unambiguously increased the fraction of women on welfare for all women. In contrast, the orthogonal estimator (Column (2)) implies that women with long history of prior AFDC receipt (at least 5 months) were pushed out of the assistance. This pattern makes sense. The Jobs First (treatment group) faced a time limit of 21 months on welfare while the AFDC (control group) had no time limit. The orthogonal estimator picks up this pattern, while the direct one fails to do so. Finally, the unpenalized model in Columns (4) and (5) finds evidence in favor of treatment effect heterogeneity, but most interaction effects are not significant at $\alpha=0.05$. Therefore, orthogonal Lasso captures a more nuanced pattern of treatment effect that is left out by direct Lasso due to a substantially larger complexity of the nuisance parameter relative to the target one, failure of the sparsity assumption (5.61), or both.

\footnotetext{
${ }^{1}$ The package is available at https://github.com/vsyrgkanis/plugin_regularized_estimation
} 
Table 1: Jobs First heterogenous effects on welfare participation

\begin{tabular}{cccccc}
\hline & $\begin{array}{c}(1) \\
\text { direct Lasso } \\
\tilde{\theta}_{\text {direct }}\end{array}$ & $\begin{array}{c}(2) \\
\text { ortho Lasso }\end{array}$ & $\begin{array}{c}(3) \\
\text { post-ortho-Lasso } \\
\hat{\theta}_{\text {PL }}\end{array}$ & $\begin{array}{c}(4) \\
\text { not penalized } \\
\hat{\theta}_{\text {unpenalized }}\end{array}$ & $\begin{array}{c}(5) \\
\text { not penalized } \\
\text { SE }\left(\hat{\theta}_{\text {unpenalized }}\right)\end{array}$ \\
\hline intercept & 0.301 & 0.528 & 0.976 & 0.035 & 2.056 \\
white & & & & 0.906 & 1.427 \\
black & & & & 1.023 & 1.456 \\
hisp & & & & 0.342 & 1.472 \\
marnvr & & & -0.395 & 1.591 \\
marapt & & & -0.167 & 1.607 \\
yrern & & & 0.000 & 0.000 \\
yrernsq & & & 0.000 & 0.000 \\
yradc & & & 0.000 & 0.000 \\
yrfst & & & 0.001 & 0.000 \\
yremp & & & -0.003 & 0.011 \\
yrvad & & & 0.006 & 0.022 \\
yrvfs & & & -0.002 & 0.020 \\
yrkvad & & & -0.115 & 0.172 \\
anyernpq & & & & 0.061 & 1.087 \\
anyadcpq & & & & 0.212 & 1.940 \\
anyfstpq & & & -0.511 & 1.938 \\
nohsged & & & 0.575 & 0.612 \\
applcant & & & 0.182 & 0.899 \\
\hline
\end{tabular}

Notes. The data come from Kline and Tartari (2016). Table reports heterogeneous Jobs First effects in the equation (5.59), estimated by the direct Lasso, ortho Lasso, postortho-Lasso-logistic, and unpenalized approaches. Each row corresponds to a treatment interaction with a covariate. The selected covariate yrkvad shows the number of months on AFDC in the year prior RA. The other covariate names are: white, black, hisp are race categories; marnvr is never married, marapt is widowed or separated; yrern/yrernsq/yradc/yrfst the total earnings/earnings squared/AFDC/food stamps in dollars the year prior to RA; yremp/yrvad/yrvfs is a binary indicator equal to one if a woman received any earnings/AFDC/food stamps in the year before RA; anyernpq/anyadcpq/anyfstpq are binary indicators equal to one if a woman received any earnings/AFDC/food stamps in her lifetime; nohsged $=1$ if the subject has no high school or GED degree and 0 otherwise; applcant $=1$ if female applied to Jobs First and 0 otherwise. Estimators: $\check{\theta}_{\text {direct }}$ as in (5.62) under assumption 5.61, the Lasso estimator $\hat{\theta}$ of Definition 4.1, post-Lasso-logistic $\hat{\theta}_{\mathrm{PL}}$, and unpenalized logistic estimator $\hat{\theta}_{\text {unpenalized }}$ defined in equation (5.64). The sample size $n=4,642$ females. For the estimation details, see text. 


\section{A. GENERAL THEORY OF EXTREMUM ESTIMATORS}

In this section, we introduce Regularized Extremum Estimator and establish its properties. Suppose there exists a population loss $Q(\theta, g)$ and its sample analog $\widehat{Q}(\theta, \widehat{g})$. Define the target parameter $\theta_{0}$ as the unique minimizer of $Q\left(\theta, g_{0}\right)$ (i.e., (2.2) holds), and the Regularized Extremum Estimator as in (2.7). In contrast to $(2.6), \widehat{Q}(\theta, \widehat{g})$ may not be a sample average. Finally, let the set $\mathbb{B}$ be as in (3.42) and the event $\mathcal{V}$ be as in (3.45).

Assumption S.1 (Convexity). With probability one, for any $g$ in the set $\mathcal{G}_{n}$, the function $\theta \rightarrow$ $\hat{Q}(\theta, g)$ is a convex twice differentiable function of $\theta$ on an open convex set that contains the parameter space $\Theta$.

Assumption S.2 (Restricted Strong Convexity on $\mathbb{B})$. There exists a constant $\bar{\gamma}>0$ so that the curvature of $Q\left(\theta, g_{0}\right)$ defined in (3.43) is bounded from below by $\bar{\gamma}$ :

$$
\inf _{\theta \in \mathbb{B}, \nu=\theta-\theta_{0}} \frac{\nu^{T} \nabla_{\theta \theta} Q\left(\theta, g_{0}\right) \nu}{\|\nu\|_{2}^{2}} \geqslant \bar{\gamma}
$$

Assumption S.3 (Uniform Convergence on $\mathbb{B})$. There exists a sequence $\tau_{n}=o(1)$ so that $\nabla_{\theta \theta} \widehat{Q}\left(\theta, g_{0}\right)$ uniformly converges to $\nabla_{\theta \theta} Q\left(\theta, g_{0}\right)$ :

$$
\sup _{\theta \in \mathbb{B}}\left\|\nabla_{\theta \theta} \widehat{Q}\left(\theta, g_{0}\right)-\nabla_{\theta \theta} Q\left(\theta, g_{0}\right)\right\|_{\infty}=O_{P}\left(\tau_{n}\right) .
$$

Assumption S.4 (Uniformly Lipschitz Hessian on $\mathbb{B})$. For any $g$ and $g^{\prime}$ in $\mathcal{G}_{n}$, there exists a sequence $\xi_{n}=o(1)$ so that the following bound holds

$$
\sup _{\theta \in \mathbb{B}}\left\|\nabla_{\theta \theta} \hat{Q}(\theta, g)-\nabla_{\theta \theta} \hat{Q}\left(\theta, g^{\prime}\right)\right\|_{\infty}=O_{P}\left(g_{n}+\xi_{n}\right) .
$$

Assumption S.5 (Convergence Rate of Empirical Gradient). For any $g \in \mathcal{G}_{n}$, there exists a sequence $\epsilon_{n}$ such that $\left\|\nabla_{\theta} \widehat{Q}\left(\theta_{0}, g\right)-\nabla_{\theta} Q\left(\theta_{0}, g\right)\right\|_{\infty}=O_{P}\left(\epsilon_{n}\right)$.

We describe the influence of the estimation error $\widehat{g}-g_{0}$ on the gradient $\nabla_{\theta} Q\left(\theta_{0}, \cdot\right)$ using pathwise derivatives w.r.t the nuisance parameter $g_{0}$. The first-order derivative is

$$
D_{r}\left[g-g_{0}, \nabla_{\theta} Q\left(\theta_{0}, g\right)\right]:=\frac{\partial}{\partial r} \nabla_{\theta} Q\left(\theta_{0}, r\left(g-g_{0}\right)+g_{0}\right)
$$

and the second-order derivative is

$$
D_{r}^{2}\left[g-g_{0}, \nabla_{\theta} Q\left(\theta_{0}, g\right)\right]:=\frac{\partial^{2}}{\partial r^{2}} \nabla_{\theta} Q\left(\theta_{0}, r\left(g-g_{0}\right)+g_{0}\right) .
$$

Assumption S.6 (Bounded Gradient of Population Loss w.r.t. Nuisance). For any $g \in \mathcal{G}_{n}$, there exist constants $B_{0}$ and $B$ so that

$\forall r \in[0,1):\left\|D_{0}\left[g-g_{0}, \nabla_{\theta} Q\left(\theta_{0}, g_{0}\right)\right]+D_{r}^{2}\left[g-g_{0}, \nabla_{\theta} Q\left(\theta_{0}, g_{0}\right)\right]\right\|_{\infty} \leqslant B_{0}\left\|g-g_{0}\right\|+B\left\|g-g_{0}\right\|^{2}$.

Theorem S.1 (Regularized Extremum Estimator). Suppose Assumptions 3.5 and S.1-S.6 hold with $k\left(\tau_{n}+g_{n}+\xi_{n}\right)=o(1)$. For $\lambda=2 C\left(\epsilon_{n}+B_{0} g_{n}+B g_{n}^{2}\right)$, and for $C$ and $n$ large enough,

$$
\left\|\hat{\theta}-\theta_{0}\right\|_{2} \lesssim_{P} \sqrt{k}\left(\epsilon_{n}+B_{0} g_{n}+g_{n}^{2}\right) \quad\left\|\hat{\theta}-\theta_{0}\right\|_{1} \lesssim_{P} k\left(\epsilon_{n}+B_{0} g_{n}+g_{n}^{2}\right) .
$$


Theorem S.1 establishes the convergence rate for Regularized Extremum Estimator. Its building blocks are described in the following lemmas.

Lemma S.1 (Convexity and Restricted Subspace, Negahban et al. (2012)). If $\frac{\lambda}{2} \geqslant\left\|\nabla_{\theta} \widehat{Q}\left(\theta_{0}, \widehat{g}\right)\right\|_{\infty}$ and $\theta \rightarrow Q(\theta, \widehat{g})$ is a convex function of $\theta$, then $\nu \in \mathcal{C}(T ; 3)$, where $\nu=\hat{\theta}-\theta_{0}$.

Proof of Lemma S.1. By definition of $\hat{\theta}$,

$$
\hat{Q}(\hat{\theta}, \widehat{g})-\widehat{Q}\left(\theta_{0}, \widehat{g}\right) \leqslant \lambda\left(\left\|\theta_{0}\right\|_{1}-\|\widehat{\theta}\|_{1}\right) .
$$

Additivity of $\ell_{1}$ norm and triangle inequality imply

$$
\begin{aligned}
\|\hat{\theta}\|_{1} & =\left\|\theta_{0}+\nu_{T}\right\|_{1}+\left\|\nu_{T^{c}}\right\|_{1} \geqslant\left\|\theta_{0}\right\|_{1}-\left\|\nu_{T}\right\|_{1}+\left\|\nu_{T^{c}}\right\|_{1} \\
\lambda\left(\left\|\theta_{0}\right\|_{1}-\|\widehat{\theta}\|_{1}\right) & \leqslant \lambda\left(\left\|\nu_{T}\right\|_{1}-\left\|\nu_{T^{c}}\right\|_{1}\right) .
\end{aligned}
$$

Convexity of $\theta \rightarrow \widehat{Q}(\theta, \widehat{g})$, Cauchy-Schwarz inequality, and the choice of $\lambda$ imply:

$$
\hat{Q}(\hat{\theta}, \widehat{g})-\hat{Q}\left(\theta_{0}, \widehat{g}\right) \geqslant \nabla_{\theta} \hat{Q}\left(\theta_{0}, \widehat{g}\right) \cdot\left(\hat{\theta}-\theta_{0}\right) \geqslant-\left\|\nabla_{\theta} \widehat{Q}\left(\theta_{0}, \widehat{g}\right)\right\|_{\infty}\|\nu\|_{1} \geqslant-\frac{\lambda}{2}\|\nu\|_{1} .
$$

Combining equations (S.6) and (S.7) gives:

$$
\lambda\left(\left\|\nu_{T}\right\|_{1}-\left\|\nu_{T^{c}}\right\|_{1}\right) \geqslant \hat{Q}(\hat{\theta}, \widehat{g})-\widehat{Q}\left(\theta_{0}, \widehat{g}\right) \geqslant-\frac{\lambda}{2}\|\nu\|_{1} .
$$

Dividing by $\lambda$ and re-arranging gives $3\left\|\nu_{T}\right\|_{1} \geqslant\left\|\nu_{T^{c}}\right\|_{1}$.

Lemma S.2 (Restricted Strong Convexity for Empirical Loss). Suppose Assumptions S.1 and S.2 hold. On the event $\mathcal{V}$, the following bound holds:

$$
\inf _{\nu \in \mathcal{C}(T ; 3)} \frac{\left(\nabla_{\theta} \widehat{Q}\left(\theta_{0}+\nu, \hat{g}\right)-\nabla_{\theta} \widehat{Q}\left(\theta_{0}, \widehat{g}\right)\right) \cdot \nu}{\|\nu\|_{2}^{2}} \geqslant \bar{\gamma} / 2 .
$$

Proof of Lemma S.2. Step 1. For some $\bar{r} \in[0,1)$ that may depend on $\hat{g}$, mean value theorem implies

$$
\left(\nabla_{\theta} \widehat{Q}\left(\theta_{0}+\nu, \widehat{g}\right)-\nabla_{\theta} \widehat{Q}\left(\theta_{0}, \widehat{g}\right)\right) \cdot \nu=\nu^{T} \cdot \nabla_{\theta \theta} \widehat{Q}\left(\theta_{0}+\bar{r} \nu, \widehat{g}\right) \cdot \nu .
$$

Invoking Cauchy-Schwartz, definition of $\mathcal{C}(T ; 3)$ in $(3.41)$, and assumption of the theorem gives:

$$
\begin{array}{lr}
\sup _{\bar{r} \in[0,1), \nu \in \mathcal{C}(T ; 3)} \frac{\left|\nu^{T} \cdot\left(\nabla_{\theta \theta} \widehat{Q}\left(\theta_{0}+\bar{r} \nu, \widehat{g}\right)-\nabla_{\theta \theta} Q\left(\theta_{0}+\bar{r} \nu, g_{0}\right)\right) \cdot \nu\right|}{\|\nu\|_{2}^{2}} & \\
\leqslant \sup _{\nu \in \mathcal{C}(T ; 3)} \frac{\|\nu\|_{1}^{2} \bar{\gamma} /(32 k)}{\|\nu\|_{2}^{2}} & \text { (Cauchy-Schwartz) } \\
\leqslant \sup _{\nu \in \mathcal{C}(T ; 3)} \frac{\left((1+3)\left\|\nu_{T}\right\|_{1}\right)^{2} \bar{\gamma} /(32 k)}{\|\nu\|_{2}^{2}} & \text { (Definition of } \mathcal{C}(T ; 3)) \\
\leqslant 16 k \bar{\gamma} /(32 k)<\bar{\gamma} / 2 . &
\end{array}
$$


Step 2. Triangle inequality implies

$$
\begin{aligned}
& \inf _{\nu \in \mathcal{C}(T ; 3)} \frac{\nu^{T} \cdot \nabla_{\theta \theta} \hat{Q}\left(\theta_{0}+\bar{r} \nu, \hat{g}\right) \cdot \nu}{\|\nu\|_{2}^{2}} \\
& \geqslant \inf _{\nu \in \mathcal{C}(T ; 3)} \frac{\nu^{T} \cdot \nabla_{\theta \theta} Q\left(\theta_{0}+\bar{r} \nu, g_{0}\right) \cdot \nu}{\|\nu\|_{2}^{2}}-\sup _{\nu \in \mathcal{C}(T ; 3)} \frac{\left|\nu^{T} \cdot\left(\nabla_{\theta \theta} \widehat{Q}\left(\theta_{0}+\bar{r} \nu, \hat{g}\right)-\nabla_{\theta \theta} Q\left(\theta_{0}+\bar{r} \nu, g_{0}\right)\right) \cdot \nu\right|}{\|\nu\|_{2}^{2}} \\
& \geqslant \bar{\gamma}-\bar{\gamma} / 2=\bar{\gamma} / 2 .
\end{aligned}
$$

Lemma S.3 (Oracle Inequality). Suppose Assumptions S.1 and S.2 hold. On the intersections of events (2.10) and (3.45), the following inequality holds:

$$
\left\|\widehat{\theta}-\theta_{0}\right\|_{2} \leqslant \frac{3 \sqrt{k}}{\bar{\gamma}} \lambda \quad\left\|\hat{\theta}-\theta_{0}\right\|_{1} \leqslant \frac{12 k}{\bar{\gamma}} \lambda .
$$

Proof of Lemma S.3. Let $\nu=\hat{\theta}-\theta_{0}$. Invoking Lemmas S.1 and S.2 gives:

$$
\begin{aligned}
& \lambda\left(\left\|\theta_{0}\right\|_{1}-\|\hat{\theta}\|_{1}\right) \geqslant \nabla_{\theta} \hat{Q}(\hat{\theta}, \hat{g}) \cdot\left(\hat{\theta}-\theta_{0}\right) \quad \text { (Optimality of } \hat{\theta} \text { ) } \\
& =\nabla_{\theta} \widehat{Q}\left(\theta_{0}, \widehat{g}\right) \cdot\left(\hat{\theta}-\theta_{0}\right)+\left(\nabla_{\theta} \widehat{Q}(\hat{\theta}, \widehat{g})-\nabla_{\theta} \widehat{Q}\left(\theta_{0}, \widehat{g}\right)\right) \cdot\left(\hat{\theta}-\theta_{0}\right) \\
& \geqslant \nabla_{\theta} \widehat{Q}\left(\theta_{0}, \hat{g}\right) \cdot\left(\hat{\theta}-\theta_{0}\right)+\frac{\bar{\gamma}}{2}\|\nu\|_{2}^{2} \\
& \geqslant-\left\|\nabla_{\theta} \widehat{Q}\left(\theta_{0}, \widehat{g}\right)\right\|_{\infty} \cdot\|\nu\|_{1}+\frac{\bar{\gamma}}{2}\|\nu\|_{2}^{2} \quad \text { (Cauchy-Schwarz) } \\
& \geqslant-\frac{\lambda}{2} \cdot\|\nu\|_{1}+\frac{\bar{\gamma}}{2}\|\nu\|_{2}^{2}
\end{aligned}
$$

Rearranging the inequality gives

$$
\frac{\bar{\gamma}}{2}\|\nu\|_{2}^{2} \leqslant \frac{3 \lambda}{2}\left\|\nu_{T}\right\|_{1}-\frac{\lambda}{2}\left\|\nu_{T^{c}}\right\|_{1} \leqslant \frac{3 \lambda}{2}\left\|\nu_{T}\right\|_{1} \leqslant \frac{3 \lambda \sqrt{k}}{2}\|\nu\|_{2} .
$$

Dividing over by $\|\nu\|_{2}$ yields the theorem. By Lemma S.1, $\nu \in \mathcal{C}(T ; 3)$ and $\|\nu\|_{1} \leqslant 4 \sqrt{k}\|\nu\|_{2}$.

Lemma S.4 (Influence on Oracle Gradient). Suppose Assumptions 3.5, S.5 and S.6 hold. With probability $1-o(1)$,

$$
\left\|\nabla_{\theta} \widehat{Q}\left(\theta_{0}, \widehat{g}\right)\right\|_{\infty}=O_{P}\left(\epsilon_{n}+B_{0} g_{n}+B g_{n}^{2}\right) .
$$

Proof of Lemma S.4. In what follows, we condition on the event $\mathcal{E}_{n}$, which holds with probability $1-o(1)$. Triangle inequality implies:

$$
\begin{aligned}
\left\|\nabla \hat{Q}\left(\theta_{0}, \widehat{g}\right)\right\|_{\infty} & \leqslant\left\|\nabla_{\theta}\left[Q\left(\theta_{0}, \widehat{g}\right)-Q\left(\theta_{0}, g_{0}\right)\right]\right\|_{\infty}+\left\|\nabla_{\theta}\left[\hat{Q}\left(\theta_{0}, \widehat{g}\right)-Q\left(\theta_{0}, \widehat{g}\right)\right]\right\|_{\infty} \\
& =I+I I .
\end{aligned}
$$

Conditional on the event $\mathcal{E}_{n}$, Assumption S.5 implies

$$
I I=\left\|\nabla_{\theta}\left[\hat{Q}\left(\theta_{0}, \hat{g}\right)-Q\left(\theta_{0}, \widehat{g}\right)\right]\right\|_{\infty}=O_{P}\left(\epsilon_{n}\right) .
$$

By Lemma 6.1 from Chernozhukov et al. (2018), this statement holds unconditionally. 
On the event $\mathcal{E}_{n}$, by Assumption S.6

$$
\begin{aligned}
I=\left\|\nabla_{\theta}\left[Q\left(\theta_{0}, \widehat{g}\right)-Q\left(\theta_{0}, g_{0}\right)\right]\right\|_{\infty} & \leqslant \sup _{g \in \mathcal{G}_{n}}\left\|\nabla_{\theta}\left[Q\left(\theta_{0}, g\right)-Q\left(\theta_{0}, g_{0}\right)\right]\right\|_{\infty} \\
& \leqslant B_{0} g_{n}+B g_{n}^{2} .
\end{aligned}
$$

Thus, (S.9) follows.

Proof of Theorem S.1. By Lemma S.4 and assumptions of the Theorem,

$$
\lambda / 2 \geqslant\left\|\nabla_{\theta} \widehat{Q}\left(\theta_{0}, \widehat{g}\right)\right\|_{\infty} \text { holds w.p. } 1-o(1) .
$$

Decomposing the difference $\nabla_{\theta \theta} \hat{Q}(\theta, \widehat{g})-\nabla_{\theta \theta} Q\left(\theta, g_{0}\right)$ gives

$$
\begin{aligned}
\sup _{\theta \in \mathbb{B}}\left\|\nabla_{\theta \theta} \hat{Q}(\theta, \hat{g})-\nabla_{\theta \theta} Q\left(\theta, g_{0}\right)\right\|_{\infty} & \leqslant \sup _{\theta \in \mathbb{B}}\left\|\nabla_{\theta \theta} \hat{Q}(\theta, \hat{g})-\nabla_{\theta \theta} \widehat{Q}\left(\theta, g_{0}\right)\right\|_{\infty} \\
& +\sup _{\theta \in \mathbb{B}}\left\|\nabla_{\theta \theta} \hat{Q}\left(\theta, g_{0}\right)-\nabla_{\theta \theta} Q\left(\theta, g_{0}\right)\right\|_{\infty} \\
& =A+B .
\end{aligned}
$$

By Assumptions 3.5, S.4, S.3, on the event $\mathcal{E}_{n}$,

$$
A=O_{P}\left(g_{n}+\xi_{n}\right), \quad B=O_{P}\left(\tau_{n}\right) .
$$

By assumption of the Theorem, $k\left(g_{n}+\xi_{n}+\tau_{n}\right)=o(1)$. Therefore, for $n$ large enough,

$$
\sup _{\theta \in \mathbb{B}}\left\|\nabla_{\theta \theta} \widehat{Q}(\theta, \widehat{g})-\nabla_{\theta \theta} Q\left(\theta, g_{0}\right)\right\|_{\infty} \leqslant \bar{\gamma} /(32 k) \text { holds w.p. } 1-o(1) .
$$

\section{B. PROOFS OF SECTION 3}

In this section, we prove Theorem 3.1 as a special case of Theorem S.1. We also prove Theorem 3.2. Define an event $\mathcal{E}_{n}:=\left\{\hat{g}_{k} \in \mathcal{G}_{n} \quad \forall k \in[K]\right\}$, such that the nuisance parameter estimate $\widehat{g}_{k}$ belongs to the realization set $\mathcal{G}_{n}$ for each fold $k \in[K]$. By union bound, this event holds w.h.p.

$$
\mathrm{P}\left(\mathcal{E}_{n}\right) \geqslant 1-K \epsilon_{n}=1-o(1) .
$$

For a given partition $k$ in $\{1,2, \ldots, K\}$, define the partition-specific averages

$$
\mathbb{E}_{n, k} f\left(W_{i}\right):=\frac{1}{n_{k}} \sum_{i \in J_{k}} f\left(W_{i}\right), \quad \mathbb{G}_{n, k} f\left(W_{i}\right):=\frac{1}{\sqrt{n_{k}}} \sum_{i \in J_{k}}\left[f\left(W_{i}\right)-\int f(w) d P(w)\right] .
$$

Lemma S.5 (Verification of Assumption S.1). Assumption 3.1 implies Assumption S.1.

Proof of Lemma S.5. If $m(w, t, \gamma)$ is non-decreasing in $t$, the loss $\ell(w, t, \gamma)$ is convex in $t$. Plugging the linear function $\theta \rightarrow t=\Lambda(z, g)^{\prime} \theta$ into the loss sketch $\ell(w, t, \gamma)$ gives the convex sample loss $\widehat{Q}(\theta, g)$.

Lemma S.6 (Verification of Assumption S.2). Assumptions 3.2 with $C_{\min }$ and 3.3 with $B_{\text {min }}$ imply Assumption S.2 with $\bar{\gamma}=B_{\min } C_{\min }$. 
Proof of Lemma S.6. Observe that

$$
\begin{aligned}
& \inf _{\theta \in \mathbb{B}} \nu^{\top} \nabla_{\theta \theta} Q\left(\theta, g_{0}\right) \nu \\
= & \inf _{\theta \in \mathbb{B}} \mathbb{E}\left[\left.\nabla_{t} m(W, t, \gamma)\right|_{\gamma=g_{0}(Z), t=\Lambda(Z, \gamma)^{\prime} \theta} \mid Z=z\right] \min \operatorname{eig} \Sigma\|\nu\|_{2}^{2} \\
& \geqslant B_{\min } C_{\min }\|\nu\|_{2}^{2} .
\end{aligned}
$$

Lemma S.7 (Verification of Assumption S.3). Lemma 3.1 verifies Assumption S.3.

Lemma S.8 (Verification of Assumption S.4). Assumptions 3.5 and 3.4 with a constant $U$ implies Assumption S.4 with $\xi_{n}=3 d \cdot U^{3} \sqrt{\frac{\log (2 p)}{n}}$ for $\mathbb{B}$ as in (3.42) and $\|g\|_{r}$ as in (3.40) for $r \in\{\infty, 2\}$.

Proof of Lemma S.8. Step 1. Observe that for each $(v, j) \in\{1,2, \ldots, p\}^{2}$,

$$
\nabla_{\theta_{v} \theta_{j}} \ell\left(w, \Lambda(z, \gamma)^{\prime} \theta, \gamma\right)=\nabla_{t} m\left(w, \Lambda(z, \gamma)^{\prime} \theta, \gamma\right) \cdot \Lambda_{v}(z, \gamma) \cdot \Lambda_{j}(z, \gamma)
$$

has its gradient in $\gamma$ bounded by $L=3 \cdot U^{3}$ in the absolute norm.

Verification of Assumption S.4 for $\|g\|=\|g\|_{\infty}$. Assumptions 3.4 implies Assumption S.4 with $L=U^{3}$.

$$
\left|\nabla_{\theta_{v} \theta_{j}} \widehat{Q}(\theta, g)-\nabla_{\theta_{v} \theta_{j}} \widehat{Q}\left(\theta, g^{\prime}\right)\right| \leqslant 3 \cdot U^{3} \sup _{z \in \mathcal{Z}}\left\|g(z)-g^{\prime}(z)\right\|_{1} .
$$

Step 2. Verification of Assumption S.4 for $\|g\|=\|g\|_{2}$. For each $(v, j) \in\{1,2, \ldots, p\}^{2}$, the function above has $U$-bounded derivative, its derivative is bounded by $L=3 U^{3}$. By McDiarmid's inequality, for any fixed $g, g^{\prime} \in \mathcal{G}_{n}$, with probability $1-o(1)$,

$$
\left|\left[\mathbb{E}_{n, k}-\mathbb{E}\right]\left[\left\|g(Z)-g^{\prime}(Z)\right\|_{1}\right]\right| \leqslant 3 d \cdot U^{3} \sqrt{\frac{\log (2 p)}{n_{k}}}=O\left(3 d \cdot U^{3} \sqrt{\frac{\log (2 p)}{n}}\right):=O\left(\xi_{n}\right) .
$$

Lemma S.9 (Verification of Assumption S.5). Assumption 3.4 with a constant $U$ implies Assumption S.5 with $\epsilon_{n}=U^{2} \cdot \sqrt{\frac{\log (2 p)}{n}}$.

Proof of Lemma S.9. Conditional on the event $\mathcal{E}_{n}$, the estimate $g=\hat{g}$ belongs to $\mathcal{G}_{n}$. Observe that

$$
\left.\nabla_{\theta_{j}} \ell\left(w, \Lambda(z, \gamma)^{\prime} \theta_{0}, \gamma\right)\right|_{\gamma=g(z)}=\left.m\left(w, \Lambda(z, \gamma)^{\prime} \theta_{0}, \gamma\right) \cdot \Lambda_{j}(z, \gamma)\right|_{\gamma=g(z)}, \quad j=1,2, \ldots p
$$

is an a.s. bounded function of data vector $w$, bounded in absolute value by $U^{2}$. By McDiarmid's inequality, the sample average of $j^{\prime}$ th function is within $U^{2} \cdot \sqrt{\frac{\log (2 p / \delta)}{n_{k}}}$ of its mean with probability at least $1-\frac{\delta}{p}$. Taking a union bound over the $p$ coordinates, we get that each coordinate is within $U^{2} \cdot \sqrt{\frac{\log (2 p / \delta)}{n_{k}}}$ from its respective mean with probability at least $1-\delta$. Therefore, $\epsilon_{n}$ can be taken to be $\epsilon_{n}=U^{2} \cdot \sqrt{\frac{\log (2 p)}{n_{k}}}=O\left(U^{2} \cdot \sqrt{\frac{\log (2 p)}{n}}\right)$. 
Lemma S.10 (Verification of Assumption S.6). Assumption 3.4 with a constant $U$ implies Assumption S.6 with $B_{0}=U^{2}$ and $B=4 U^{2}$.

Proof of Lemma S.10. By Assumption 3.4, for any $w, \theta$ and $g \in \mathcal{G}_{n}$, define the matrix $A_{j} \in$ $\mathrm{R}^{d \times d}$ as

$$
A_{j}:=A_{j}(w, \theta, g)=\nabla_{\gamma \gamma}\left[\left.m(w, t, \gamma)\right|_{\gamma=g(z), t=\Lambda(z, \gamma)^{\prime} \theta} \cdot \Lambda_{j}(z, \gamma)\right] .
$$

By Cauchy-Schwarz inequality,

$$
\left|\left(g(Z)-g_{0}(Z)\right)^{\prime} A_{j}\left(g(Z)-g_{0}(Z)\right)\right| \leqslant\left\|A_{j}\right\|_{\infty}\left\|\left(g(Z)-g_{0}(Z)\right)\right\|_{1}^{2} .
$$

Taking expectations on each side and invoking $\sup _{1 \leqslant j \leqslant p}\left\|A_{j}\right\|_{\infty} \leqslant 4 \cdot U^{2} \quad$ a.s. gives

$$
\begin{aligned}
D_{r}^{2}\left[g-g_{0}, \nabla_{\theta_{j}} Q\left(\theta_{0}, g_{0}\right)\right] & \leqslant \mathbb{E}\left|\left(g(Z)-g_{0}(Z)\right)^{\prime} A_{j}\left(g(Z)-g_{0}(Z)\right)\right| \leqslant 4 \cdot U^{2} \cdot \mathbb{E}\left[\left\|g(Z)-g_{0}(Z)\right\|_{1}^{2}\right] \\
& \leqslant 4 \cdot U^{2} \sup _{z \in \mathcal{Z}}\left\|g(z)-g_{0}(z)\right\|_{1}^{2} \\
& \leqslant 4 \cdot U^{2}\left\|g-g_{0}\right\|_{\infty}^{2}
\end{aligned}
$$

Likewise, $\left\|D_{0}\left[g-g_{0}, \nabla_{\theta} Q\left(\theta_{0}, g_{0}\right)\right]\right\|_{\infty} \leqslant U^{2} \mathbb{E}\left[\left\|g(Z)-g_{0}(Z)\right\|_{1}^{2}\right.$.

Proof of Theorem 3.1. Theorem 3.1 follows from the statement of Theorem S.1 and Lemmas S.5-S.10.

Proof of Theorem 3.2. Step 1. The validity of the adjusted CMR can be seen from

$$
\begin{aligned}
\mathbb{E}\left[\left.m\left(W, \Lambda\left(Z, \gamma_{1}\right)^{\prime} \theta_{0}, \gamma\right)\right|_{\gamma=g_{0}(Z)} \mid Z=z\right] & =\mathbb{E}\left[\left.m_{\text {pre }}\left(W, \Lambda\left(Z, \gamma_{1}\right)^{\prime} \theta_{0}, \gamma_{1}\right)\right|_{\gamma_{1}=p_{0}(Z)} \mid Z=z\right] \\
& -h_{0}(z) I_{0}^{-1}(z) \mathbb{E}\left[R\left(W, p_{0}(Z)\right) \mid Z=z\right]=0,
\end{aligned}
$$

which follows from (3.47) and (3.48).

Step 2. We verify the orthogonality condition (2.8) in two steps. First, we compute the derivative of (2.5) with respect to $\gamma=\left(\gamma_{1}, \gamma_{2}, \gamma_{3}\right)$

$$
\begin{aligned}
& \mathbb{E}\left[\left.\nabla_{\gamma} m\left(W, \Lambda\left(z, \gamma_{1}\right)^{\prime} \theta_{0}, \gamma\right) \cdot \Lambda\left(z, \gamma_{1}\right)\right|_{\gamma=g_{0}(z)} \mid Z=z\right] \\
+ & \mathbb{E}\left[\left.m\left(W, \Lambda\left(z, \gamma_{1}\right)^{\prime} \theta_{0}, \gamma\right) \cdot \nabla_{\gamma} \Lambda\left(z, \gamma_{1}\right)\right|_{\gamma=g_{0}(z)} \mid Z=z\right] \\
& =(0,0,0)^{\prime}+(0,0,0)^{\prime}=i+i i,
\end{aligned}
$$

where $i$ is shown in Step 3 and $i$ follows from Step 1.

Step 3. The partial derivatives with respect to $\gamma=\left(\gamma_{1}, \gamma_{2}, \gamma_{3}\right)$ are mean zero conditionally on $z$.

$$
\begin{aligned}
& \mathbb{E}\left[\left.\nabla_{\gamma_{1}} m\left(W, \Lambda\left(z, \gamma_{1}\right)^{\prime} \theta_{0}, \gamma\right)\right|_{\gamma=g_{0}(z)} \mid Z=z\right] \\
& =\mathbb{E}\left[\nabla_{\gamma_{1}} m_{\mathrm{pre}}\left(W, \Lambda\left(z, \gamma_{1}\right)^{\prime} \theta_{0}, \gamma_{1}\right) \mid Z=z\right]-h_{0}(z) I_{0}(z)^{-1} I_{0}(z)=0 .
\end{aligned}
$$




$$
\begin{gathered}
\text { Regularized Machine Learning } \\
\mathbb{E}\left[\left.\nabla_{\gamma_{2}} m\left(W, \Lambda\left(z, \gamma_{1}\right)^{\prime} \theta_{0}, \gamma\right)\right|_{\gamma=g_{0}(z)} \mid Z=z\right]=-I_{0}(z)^{-1} \mathbb{E}\left[R\left(W, p_{0}(Z)\right) \mid Z=z\right]=0 \\
\mathbb{E}\left[\left.\nabla_{\gamma_{3}} m\left(W, \Lambda\left(z, \gamma_{1}\right)^{\prime} \theta_{0}, \gamma\right)\right|_{\gamma=g_{0}(z)} \mid Z=z\right]=h_{0}(z) I_{0}^{-2}(z) \mathbb{E}\left[R\left(W, p_{0}(Z)\right) \mid Z=z\right]=0
\end{gathered}
$$

Step 4. Verification of Assumptions 3.1-3.4 for the moment function $m(w, t, \gamma)$ in (3.49). First, observe that

$$
h_{0}(z) I_{0}^{-1}(z) R\left(W, p_{0}(Z)\right)
$$

does not depend on the single index $t$. Therefore, the moment function $m(w, t, \gamma)$ is monotone in $t$ if and only if $m_{\text {pre }}\left(w, t, \gamma_{1}\right)$ is monotone in $t$. Likewise, $m(w, t, \gamma)$ is single index in $t$ if and only if $m_{\text {pre }}\left(w, t, \gamma_{1}\right)$ is single index in $t$. Finally,

$$
\nabla_{t} m(w, t, \gamma)=\nabla_{t} m_{\text {pre }}\left(w, t, \gamma_{1}\right)
$$

for any value of $w, t, \gamma$. The function $(w, \gamma) \rightarrow \gamma_{2} \gamma_{3}^{-1} R\left(w, \gamma_{1}\right)$ is a smooth function of $\gamma=\left(\gamma_{1}, \gamma_{2}, \gamma_{3}\right)$ by assumption of the theorem, implying Assumption 3.4.

\section{PROOFS OF SECTION 4}

Let $\check{\theta}$ be a preliminary estimator of $\theta_{0}$ converging at rate $\theta_{n}$. Lemma S.11 establishes the shrinkage rates of the nuisance realization sets introduced below.

1 Suppose

$$
R_{h}\left(z, t, \gamma_{1}\right)=\mathbb{E}\left[\nabla_{\gamma_{1}} m_{\text {pre }}\left(W, t, \gamma_{1}\right) \mid Z=z\right]
$$

is a known function of $z, t, \gamma_{1}$. Define the realization set $\mathcal{H}_{n}=\mathcal{H}_{n, r}$ for $r \in\{2, \infty\}$

$$
\mathcal{H}_{n}=\left\{R_{h}\left(z, \Lambda(z, p(z))^{\prime} \theta, p(z)\right):\left\|p-p_{0}\right\|_{r} \leqslant p_{n},\left\|\theta-\theta_{0}\right\|_{1} \leqslant \theta_{n}\right\} .
$$

2 Suppose

$$
R_{I}\left(z, \gamma_{1}\right)=\mathbb{E}\left[\nabla_{\gamma_{1}} R\left(W, \gamma_{1}\right) \mid Z=z\right]
$$

is a known function of $z, \gamma_{1}$. Define the realization set $I_{n}=I_{n, r}$ for $r \in\{2, \infty\}$ as

$$
I_{n}=\left\{R_{I}(w, p(z)):\left\|p-p_{0}\right\|_{r} \leqslant \pi_{n}\right\}
$$

3 Suppose $m_{\text {pre }}\left(w, t, \gamma_{1}\right)$ is a known function of $w, t, \gamma_{1}$ and let

$$
q_{0}\left(z, t, \gamma_{1}\right)=\mathbb{E}\left[m_{\text {pre }}\left(W, t, \gamma_{1}\right) \mid Z=z\right] .
$$

Define the realization set as

$$
\mathcal{H}_{n}\left(q_{0}\right)=\left\{q(z, t, p(z)): \sup _{t \in \mathbb{R}} \sup _{\gamma \in \Gamma}\left\|q(\cdot, t, \gamma)-q_{0}(\cdot, t, \gamma)\right\|_{r} \leqslant q_{n},\left\|\theta-\theta_{0}\right\|_{1} \leqslant \theta_{n},\left\|p-p_{0}\right\|_{r} \leqslant \pi_{n}\right\}
$$

Lemma S.11 (Plausibility of Assumption 3.5 for the adjusted CMR (3.49)). Suppose Assumptions 3.1-3.4 hold with $U \geqslant 1$.

1 The plug-in estimator $\widehat{h}(z)$

$$
\widehat{h}(z):=R_{h}\left(z, \Lambda(z, \widehat{p}(z))^{\prime} \check{\theta}, \widehat{p}(z)\right)
$$


of $h_{0}(z)$ belongs to $\mathcal{H}_{n}$ w.p. $1-o(1)$. The set $\mathcal{H}_{n}$ shrinks at rate $h_{n, r}=U^{2}\left(\theta_{n}+\pi_{n, r}\right)$ for $r=\infty$ and $h_{n, 2}=\sqrt{2} U^{2}\left(\theta_{n}+\pi_{n, 2}\right)$.

2 The plug-in estimator $\widehat{I}(z)$

$$
\widehat{I}(z)=R_{I}(z, \widehat{p}(z))
$$

of $I_{0}(z)$ belongs to $I_{n}$ w.p. $1-o(1)$. The set $I_{n}$ shrinks at rate $i_{n, r}=U \pi_{n, r}$ for $r \in\{\infty, 2\}$.

3 The estimator $\hat{h}(z)$ of $h_{0}(z)$

$$
\widehat{h}(z):=\widehat{q}\left(z, \Lambda(z, \widehat{p}(z))^{\prime} \check{\theta}, \widehat{p}(z)\right)
$$

belongs to the realization set $\mathcal{H}_{n}\left(q_{0}\right)=\mathcal{H}_{n, r}\left(q_{0}\right)$ for $r \in\{2, \infty\}$ w.p. $1-o(1)$. The set $\mathcal{H}_{n}\left(q_{0}\right)$ shrinks at rate $h_{n, r}=U^{2}\left(\theta_{n}+\pi_{n, r}\right)+q_{n, r}$ for $r=\infty$ and $h_{n, 2}=2\left(U^{2}\left(\theta_{n}+\right.\right.$ $\left.\left.\pi_{n, 2}\right)+q_{n, 2}\right)$.

Proof of Lemma S.11. Step 1. Proof of Lemma S.11 (1). By Assumption 3.4, $m_{\text {pre }}\left(w, t, \gamma_{1}\right)$ and $\Lambda\left(z, \gamma_{1}\right)$ are smooth functions of $t$ and $\gamma_{1}$. Therefore, $R_{h}\left(z, t, \gamma_{1}\right)$ is a smooth function of $t$ and $\gamma_{1}$ whose partial derivatives are $U$-bounded uniformly over all arguments. By intermediate value theorem,

$$
\begin{aligned}
R_{h}(z, t, \gamma)-R_{h}\left(z, t_{0}, \gamma_{0}\right) & =\nabla_{t} R_{h}(z, \bar{t}, \gamma) \Lambda(z, \gamma)^{\prime}\left(\theta-\theta_{0}\right)+\nabla_{\gamma} R_{h}\left(z, \Lambda(z, \bar{\gamma})^{\prime} \theta_{0}, \bar{\gamma}\right) \cdot\left(\gamma-\gamma_{0}\right) \\
& =I_{1}(z)+I_{2}(z) .
\end{aligned}
$$

By Cauchy Schwartz and Assumption 3.4, for any $\theta:\left\|\theta-\theta_{0}\right\|_{1} \leqslant \theta_{n}$,

$$
\sup _{z \in \mathcal{Z}}\left|I_{1}(z)\right| \leqslant U \sup _{z \in \mathcal{Z}} \sup _{\gamma \in \Gamma}\left|\Lambda(z, \gamma)^{\prime}\left(\theta-\theta_{0}\right)\right| \leqslant U^{2}\left\|\theta-\theta_{0}\right\|_{1} \leqslant U^{2} \theta_{n} .
$$

Plugging $\gamma=p(z)$ and $\gamma_{0}=p_{0}(z)$ gives the bound in $\ell_{\infty}$ norm:

$$
\sup _{z \in \mathcal{Z}}\left|I_{1}(z)+I_{2}(z)\right| \leqslant U^{2} \theta_{n}+U \pi_{n} \leqslant U^{2}\left(\theta_{n}+\pi_{n}\right) .
$$

Therefore, $\mathcal{H}_{n}$ shrinks at rate $h_{n}=U^{2}\left(\theta_{n}+\pi_{n}\right)$ for $\pi_{n}=\pi_{n, \infty}$.

In $\ell_{2}$ norm, the bound is

$$
\left(\mathbb{E}\left(I_{1}(Z)+I_{2}(Z)\right)^{2}\right)^{1 / 2} \leqslant \sqrt{2}\left(\left(\mathbb{E} I_{1}^{2}(Z)\right)^{1 / 2}+\left(\mathbb{E} I_{2}^{2}(Z)\right)^{1 / 2}\right) \leqslant \sqrt{2} U^{2}\left(\theta_{n}+\pi_{n}\right)
$$

for $\pi_{n}=\pi_{n, 2}$.

Step 2. Proof of Lemma S.11 (2). By intermediate value theorem, $I_{n}$ in $\ell_{r}$ norm shrinks at rate $i_{n}=U \pi_{n}$ if $\mathcal{P}_{n}$ shrinks at rate $\pi_{n, r}$, for $r \in\{2, \infty\}$.

Step 3. Proof of Lemma S.11 (3). Observe that

$$
\begin{aligned}
q(z, t, p(z))-q_{0}\left(z, t_{0}, p_{0}(z)\right) & =\left(q(z, t, p(z))-q_{0}(z, t, p(z))\right)+\left(q_{0}(z, t, p(z))-q_{0}\left(z, t_{0}, p_{0}(z)\right)\right) \\
& =Q_{1}(z)+Q_{2}(z) .
\end{aligned}
$$

Consider the case $r=\infty$. The bound on $Q_{1}(z)$ in $\ell_{\infty}$ follows from

$$
\sup _{z \in \mathcal{Z}}\left|Q_{1}(z)\right| \leqslant \sup _{t \in \mathbb{R}} \sup _{\gamma \in \Gamma}\left|q(z, t, \gamma)-q_{0}(z, t, \gamma)\right| \leqslant q_{n} .
$$

Invoking Step 1 with $R_{h}(z, t, \gamma)=q_{0}(z, t, \gamma)$ gives

$$
\sup _{z \in \mathcal{Z}}\left|Q_{2}(z)\right| \leqslant U^{2}\left(\theta_{n}+\pi_{n}\right) \text {. }
$$

Consider the case $r=2$. The bound on $Q_{1}(z)$ in $\ell_{2}$ follows from

$$
\left(\mathbb{E} Q_{1}^{2}(Z)\right)^{1 / 2} \leqslant \sup _{t \in \mathbb{R}} \sup _{\gamma \in \Gamma}\left(\mathbb{E}\left(q(Z, t, \gamma)-q_{0}(Z, t, \gamma)\right)^{2}\right)^{1 / 2} \leqslant q_{n} .
$$


Invoking Step 1 with $R_{h}(z, t, \gamma)=q_{0}(z, t, \gamma)$ gives

$$
\left(\mathbb{E} Q_{2}^{2}(Z)\right)^{1 / 2} \leqslant \sqrt{2} U^{2}\left(\theta_{n}+\pi_{n}\right) .
$$

Proof of Corollary 4.1. Step 1. Assumption 3.2 is a restatement of Assumption 4.6 (1). Assumption 3.3 follows from Assumption 4.6 (2). Indeed, for any $w \in \mathcal{W}$ and $\nu \in \mathcal{C}(T ; 3)$ the following bound holds:

$$
\left|\left(d-p_{0}(x)\right) \cdot(1, x)^{\prime}\left(\theta_{0}+\nu\right)+q_{0}(x)\right| \leqslant H_{\mathrm{TE}}^{2}\left\|\theta_{0}+\nu\right\|_{1}+H_{\mathrm{TE}} \leqslant 2 H_{\mathrm{TE}}^{3} .
$$

Therefore,

$$
\inf _{\theta \in \mathbb{B}} \nabla_{t} \mathbb{E}\left[m\left(W, \Lambda\left(Z, g_{0}(Z)\right)^{\prime} \theta, g_{0}(Z)\right) \mid Z=z\right]=\inf _{\theta \in \mathbb{B}} \frac{G^{\prime}\left(\left(d-p_{0}(x)\right) \cdot(1, x)^{\prime} \theta+q_{0}(x)\right)}{V_{0}(z)} \geqslant U_{\mathrm{TE}} \mathrm{B}_{\min } .
$$

Assumption 3.1 follows from Assumption 4.6 (4).

Step 2. Verification of Assumption 3.4. Let $x_{0}=1$. For each $j \in\{0,1,2, \ldots, p-1\}$ the function $\Lambda_{j}\left(z, \gamma_{1}\right)=\left(d-\gamma_{1}\right) \cdot x_{j}$ is bounded by $H_{\mathrm{TE}}^{2}$ for any $w \in \mathcal{W}$ and $\gamma_{1} \in \Gamma_{1}$. The first derivative $\nabla_{\gamma_{1}} \Lambda_{j}\left(z, \gamma_{1}\right)=x_{j}$ is bounded by $H_{\mathrm{TE}}$ a.s. Finally, the second derivative $\nabla_{\gamma_{1} \gamma_{1}} \Lambda_{j}\left(z, \gamma_{1}\right)=0$ is zero. Next, the function $t \rightarrow G(t)-y$ is 3-times differentiable in $t$ with derivatives bounded by $U_{\mathrm{TE}}$. The first (second) derivative of $\gamma_{3}^{-1}$ is bounded by $U_{\mathrm{TE}}\left(2 U_{\mathrm{TE}}\right)$, respectively. Since the moment function $m(w, t, \gamma)$ is a product of $G(t+$ $\left.\gamma_{2}\right)-y$ and $\gamma_{3}^{-1}$, all the quantities in Assumption 3.4 are bounded by $U=C_{U_{\mathrm{TE}}} U_{\mathrm{TE}}^{2}$ for a sufficiently large absolute constant $C_{U_{\mathrm{TE}}}$.

Step 3. Verification of Assumption 3.5. For $r \in\{2, \infty\}$, let $\pi_{n}=\pi_{n, r}$ and $q_{n}=q_{n, r}$. By Theorem 3.1, Step 1 of Definition 4.1 converges in $\ell_{1}$-norm with $\theta_{n}=k C_{\operatorname{TE}}\left(\sqrt{\frac{\log p}{n}}+\pi_{n}+q_{n}\right)$ for a sufficiently large $C_{\mathrm{TE}}$. By Lemma S.11(1), the estimate $\hat{V}(d, x)$ in (4.54) converges at rate $C_{U_{\mathrm{TE}}} U_{\mathrm{TE}}^{2}\left(\pi_{n}+q_{n}+\theta_{n}\right)$ in $\ell_{r}$ norm. Then, Assumption 3.5 holds with $g_{n}=C_{g} k\left(\pi_{n}+q_{n}+\sqrt{\frac{\log p}{n}}\right)$ for a sufficiently large absolute constant $C_{g}$.

Step 4. Verification of orthogonality condition (2.8). We show that the loss function gradient (2.5) is an orthogonal moment equation with respect to perturbations of $g_{0}(z)=\left\{p_{0}(x), q_{0}(x), V_{0}(d, x)\right\}$.

$$
\begin{aligned}
& \frac{\partial}{\partial r} \nabla_{\theta} Q\left(\theta_{0}, r\left(p-p_{0}\right)+p_{0}\right)=-\mathbb{E}\left(D-p_{0}(X)\right)\left(p(X)-p_{0}(X)\right) \cdot(1, X)^{\prime} \\
& -\frac{1}{V_{0}(D, X)} \mathbb{E}\left(Y-G\left(\left(D-p_{0}(X)\right) \cdot\left((1, X)^{\prime} \theta_{0}\right)+q_{0}(X)\right)\right)\left(p(X)-p_{0}(X)\right) \cdot(1, X)^{\prime}=0 \\
& \frac{\partial}{\partial r} \nabla_{\theta} Q\left(\theta_{0}, r\left(q-q_{0}\right)+q_{0}\right)=-\mathbb{E}\left(D-p_{0}(X)\right)\left(q(X)-q_{0}(X)\right) \cdot(1, X)^{\prime}=0 \\
& \frac{\partial}{\partial r} \nabla_{\theta} Q\left(\theta_{0}, r\left(V-V_{0}\right)+V_{0}\right)=-\frac{1}{V_{0}^{2}(D, X)} \mathbb{E}\left(Y-G\left(\left(D-p_{0}(X)\right) \cdot\left((1, X)^{\prime} \theta_{0}\right)+q_{0}(X)\right)\right) . \\
& \left(D-p_{0}(X)\right)\left(V(D, X)-V_{0}(D, X)\right) \cdot(1, X)^{\prime}=0 .
\end{aligned}
$$

Proof of Corollary 4.2. The loss function (2.32) in Example 2.2 is a special case of (3.52). By Theorem 3.2, the population gradient based on (2.32) obeys the orthogonality condition (2.8). 
Step 1. Assumption 3.2 holds by Assumption 4.7 (1). Assumption 3.3 holds by

$$
\inf _{\theta \in \mathbb{B}} \nabla_{t} \mathbb{E}\left[m\left(W, \Lambda\left(Z, g_{0}(Z)\right)^{\prime} \theta, g_{0}(Z)\right) \mid Z=z\right]=\inf _{t \in \mathcal{T}} \nabla_{t} \mathbb{E}[u(Y, t) \mid X=x] \geqslant B_{\min }
$$

for any $x \in \mathcal{X}$. Assumption 3.1 directly follows from Assumption 4.7 (5).

Step 2. Verification of Assumption 3.4. Observe that $\Lambda(x, \gamma)=x$ and $\|x\|_{\infty} \leqslant H_{\mathrm{MD}}$. Next, the function $t \rightarrow v \cdot u(y, t)$ is 3-times differentiable in $t$ with derivatives bounded by $U_{\mathrm{MD}}$. The second derivative of $\gamma_{1}^{-1}$ on $\Gamma_{1}$ is bounded by $6 \bar{p}^{-3}$. Since the moment function $m_{\text {pre }}\left(w, t, \gamma_{1}\right)$ is a product of $v \cdot u(y, t)$ and $\gamma_{1}^{-1}$, all the quantities in Assumption 3.4 are bounded by $C_{\mathrm{MD}}\left(U_{\mathrm{MD}}^{2}+6 \bar{p}^{-3}\right)$ for a sufficiently large absolute constant $C_{\mathrm{MD}}$.

Step 3. Verification of Assumption 3.5. For $r \in\{2, \infty\}$, let $\pi_{n}=\pi_{n, r}$ and $q_{n}=q_{n, r}$. By Theorem 3.1, Step 1 of Definition 4.1 converges in $\ell_{1}$-norm with $\theta_{n}=k C_{\mathrm{MD}}\left(\sqrt{\frac{\log p}{n}}+\pi_{n}\right)$ for a sufficiently large $C_{\mathrm{MD}}$. By Lemma S.11(3), the estimate $\hat{h}(x)$ in Remark 4.6 converges at rate $C_{\mathrm{MD}}\left(\pi_{n}+\theta_{n}\right)+q_{n}$. Thus, Assumption 3.5 holds with

$$
g_{n}=C_{g}\left(k\left(\pi_{n}+\sqrt{\frac{\log p}{n}}\right)+q_{n}\right)
$$

for a sufficiently large absolute constant $C_{g}$.

Proof of Corollary 4.3. The loss function (2.32) in Example 2.3 is a special case of (3.52). By Theorem 3.2, the population gradient based on (2.32) obeys the orthogonality condition (2.8).

Step 1. Assumption 3.2 holds by Assumption 4.8 (1). Assumption 3.3 holds by

$$
\inf _{\theta \in \mathcal{B}} \nabla_{t} \mathbb{E}\left[m\left(W, \Lambda\left(Z, g_{0}(Z)\right)^{\prime} \theta, g_{0}(Z)\right) \mid Z=z\right]=\inf _{t \in \mathcal{T}} \mathcal{L}(t) \geqslant B_{\min }
$$

for any $x \in \mathcal{X}$. Assumption 3.1 follows from the monotonicity of logistic function.

Step 2. Verification of Assumption 3.4. Observe that $\Lambda(x, \gamma)=\left(x ; \gamma_{1}\right),\|x\|_{\infty} \leqslant H_{\text {games }}$, and $\gamma_{1} \in[0,1]$. Next, the function $t \rightarrow-(y-\mathcal{L}(t))$ is 3-times differentiable in $t$ with derivatives bounded by some constant $U_{\text {games }}$, which can be taken $U_{\text {games }} \geqslant H_{\text {games }}$. Therefore, all the quantities in Assumption 3.4 are bounded by $C_{U_{\text {games }}} U_{\text {games }}$ for a sufficiently large absolute constant $C_{U_{\text {games }}}$.

Step 3. Verification of Assumption 3.5. For $r \in\{2, \infty\}$, let $\pi_{n}=\pi_{n, r}$. By Theorem 3.1, Step 1 of Definition 4.1 converges in $\ell_{1}$-norm with $\theta_{n}=k C_{\text {games }}\left(\sqrt{\frac{\log p}{n}}+\pi_{n}\right)$ for a sufficiently large $C_{\text {games }}$. By Lemma S.11(1), the estimate $\widehat{h}(x)$ in Remark 4.7 converges at rate $C_{\text {games }}\left(\pi_{n}+\theta_{n}\right)$. Thus, Assumption 3.5 holds with

$$
g_{n}=C_{g}\left(k\left(\pi_{n}+\sqrt{\frac{\log p}{n}}\right)\right)
$$

for a sufficiently large absolute constant $C_{g}$.

\section{REFERENCES}

Ai, C. and X. Chen (2003). Efficient estimation of models with conditional moment restrictions containing unknown functions. Econometrica 71(6), 1795-1843. 
Andrews, D. (1994). Asymptotics for semiparametric econometric models via stochastic equicontinuity. Econometrica 62(1), 43-72.

Bajari, P., H. Hong, J. Krainer, and D. Nekipelov (2010). Estimating static models of strategic interactions. Journal of Business \& Economic Statistics 28(4), 469-482.

Bajari, P., H. Hong, and D. Nekipelov (2013). Game theory and econometrics: A survey of some recent research. In Advances in economics and econometrics, 10th world congress, Volume 3, pp. 3-52.

Belloni, A. and V. Chernozhukov (2013). Least squares after model selection in highdimensional sparse models. Bernoulli (19), 521-547.

Belloni, A., V. Chernozhukov, et al. (2011). $\ell_{1}$-penalized quantile regression in highdimensional sparse models. The Annals of Statistics 39(1), 82-130.

Belloni, A., V. Chernozhukov, D. Chetverikov, C. Hansen, and K. Kato (2018, June). High-dimensional econometrics and regularized GMM. arXiv e-prints, arXiv:1806.01888.

Belloni, A., V. Chernozhukov, and Y. Wei (2016). Post-selection inference for generalized linear models with many controls. Journal of Business and Economic Statistics 36, $606-$ 619.

Bickel, P. J., Y. Ritov, J. Klaassen, and J. A. Wellner (1993). Efficient and adaptive estimation for semiparametric models. Johns Hopkins University Press Baltimore.

Breiman, L. (2001). Random forests. Machine Learning 45, 5-32.

Carroll, R. J., D. Ruppert, and L. A. Stefanski (1995). Measurement error in nonlinear models. number 63 in monographs on statistics and applied probability.

Carroll, R. J. and M. Wand (1991). Semiparametric estimation in logistic measurement error models. Journal of the Royal Statistical Society. Series B (Methodological), 573-585.

Chakrabortty, A., J. Lu, T. T. Cai, and H. Li (2019, November). High Dimensional MEstimation with Missing Outcomes: A Semi-Parametric Framework. arXiv e-prints, arXiv:1911.11345.

Chen, X., H. Hong, and A. Tarozzi (2008). Semiparametric efficiency in gmm models with auxiliary data. The Annals of Statistics 36(2), 808-843.

Chen, $X$. and H. White (1999). Improved rates and asymptotic normality for nonparametric neural network estimators. IEEE Transactions on Information Theory, 682-691.

Chernozhukov, V., D. Chetverikov, M. Demirer, E. Duflo, C. Hansen, W. Newey, and J. Robins (2018). Double/debiased machine learning for treatment and structural parameters. Econometrics Journal 21, C1-C68.

Chernozhukov, V., J. C. Escanciano, H. Ichimura, W. K. Newey, and J. M. Robins (2016, July). Locally Robust Semiparametric Estimation. arXiv e-prints, arXiv:1608.00033.

Chernozhukov, V., W. Newey, and R. Singh (2018a, February). De-biased machine learning of global and local parameters using regularized riesz representers. arXiv e-prints, arXiv:1802.08667.

Chernozhukov, V., W. K. Newey, and R. Singh (2018b, September). Automatic debiased machine learning of causal and structural effects. arXiv e-prints, arXiv:1809.05224.

Chiang, H. D. (2018, December). Many Average Partial Effects: with An Application to Text Regression. arXiv e-prints, arXiv:1812.09397.

Chiang, H. D., K. Kato, Y. Ma, and Y. Sasaki (2019, September). Multiway Cluster Robust Double/Debiased Machine Learning. arXiv e-prints, arXiv:1909.03489.

Colangelo, K. and Y.-Y. Lee (2020, April). Double Debiased Machine Learning Nonparametric Inference with Continuous Treatments. arXiv e-prints, arXiv:2004.03036.

Efron, B., T. Hastie, and R. Tibshirani (2007). Discussion: The dantzig selector: Statistical estimation when $p$ is much larger than $n$. The Annals of Statistics 35(6), 2358-2364. 
Fan, Q., Y.-C. Hsu, R. P. Lieli, and Y. Zhang (2019, August). Estimation of conditional average treatment effects with high-dimensional data. arXiv e-prints, arXiv:1908.02399.

Foster, D. J. and V. Syrgkanis (2019, January). Orthogonal statistical learning. arXiv e-prints, arXiv:1901.09036.

Hardle, W., P. Hall, and H. Ichimura (1993). Optimal smoothing in single-index models. Annals of Statistics 21(1), 157-178.

Hardle, W. and T. Stoker (1989). Investigating smooth multiple regression by the method of average derivatives. Journal of American Statistical Association 84(408), 986-995.

Ichimura, H. (1993). Semiparametric least squares (sls) and weighted sls estimation of single-index models. Journal of Econometrics 58, 71-120.

Ichimura, H. and W. K. Newey (2015, August). The Influence Function of Semiparametric Estimators. arXiv e-prints, arXiv:1508.01378.

Jeong, S. and H. Namkoong (2020, July). Robust causal inference under covariate shift via worst-case subpopulation treatment effects. arXiv e-prints, arXiv:2007.02411.

Klein, R. and R. Spady (1993). An efficient semiparametric estimator for binary response models. Econometrica 61(2), 387-421.

Kline, P. and M. Tartari (2016). Bounding the labor supply responses to a randomized welfare experiment: a revealed preference approach. American Economic Review 106(4), 972-1014.

Lee, L.-F. and J. H. Sepanski (1995). Estimation of linear and nonlinear errors-invariables models using validation data. Journal of the American Statistical Association 90(429), 130-140.

Leeb, H. and B. Potcher (2005). Model selection and inference: Facts and fiction. Econometric Theory 21, 21-59.

Loh, P.-L. (2017, 04). Statistical consistency and asymptotic normality for highdimensional robust $m$-estimators. Ann. Statist. 45(2), 866-896.

Loh, P.-L. and M. J. Wainwright (2013). Regularized m-estimators with nonconvexity: Statistical and algorithmic theory for local optima. In Advances in Neural Information Processing Systems, pp. 476-484.

Luo, Y. and M. Spindler (2016, February). High-dimensional $L_{2}$-boosting: rate of convergence. arXiv e-prints, arXiv:1602.08927.

Malley, J., J. Kruppa, A. Dasgupta, K. Malley, and A. Ziegler (2012). Consistent probability estimation using nonparametric learning machines. Methods of Information in Medicine 51, 74-81.

Manski, C. F. (1975). Maximum score estimation of the stochastic utility model of choice. Journal of Econometrics 3, 205-208.

Manski, C. F. (1985). Semiparametric analysis of discrete response: asymptotic properties of the maximum score estimator. Journal of Econometrics 27, 313-333.

Negahban, S. N., P. Ravikumar, M. J. Wainwright, and B. Yu (2012, Nov). A unified framework for high-dimensional analysis of $m$-estimators with decomposable regularizers. Statistical Science 27(4), 538-557.

Newey, W. (1994, November). The asymptotic variance of semiparametric estimators. Econometrica 62(6), 245-271.

Newey, W. and T. Stoker (1993, September). Efficiency of weighted average derivative estimators and index models. Econometrica 61(5), 1199-1223.

Neyman, J. (1959). Optimal asymptotic tests of composite statistical hypotheses. Probability and Statistics 213(57), 416-444.

Neyman, J. (1979). $c(\alpha)$ tests and their use. Sankhya, 1-21. 
Nie, X. and S. Wager (2017, December). Quasi-oracle estimation of heterogeneous treatment effects. arXiv e-prints, arXiv:1712.04912.

Oprescu, M., V. Syrgkanis, and Z. S. Wu (2018, June). Orthogonal random forest for causal inference. arXiv e-prints, arXiv:1806.03467.

Powell, J. L. (1984). Least absolute deviations estimation for the censored regression model. Journal of Econometrics 27, 303-325.

Robins, J. and A. Rotnitzky (1995). Semiparametric efficiency in multivariate regression models with missing data. Journal of American Statistical Association 90(429), 122-129.

Robinson, P. M. (1988, July). Root-n-consistent semiparametric regression. Econometrica 56(4), 931-954.

Sasaki, Y. and T. Ura (2018, May). Estimation and inference for Policy Relevant Treatment Effects. arXiv e-prints, arXiv:1805.11503.

Sasaki, Y., T. Ura, and Y. Zhang (2020, July). Unconditional quantile regression with high-dimensional data. arXiv e-prints, arXiv:2007.13659.

Semenova, V. and V. Chernozhukov (2020). Debiased machine learning of conditional average treatment effect and other causal functions. Econometrics Journal (Forthcoming).

Semenova, V., M. Goldman, V. Chernozhukov, and M. Taddy (2017, December). Estimation and inference about heterogeneous treatment effects in high-dimensional dynamic panels. arXiv e-prints, arXiv:1712.09988.

Sepanski, J. H. and R. J. Carroll (1993). Semiparametric quasilikelihood and variance function estimation in measurement error models. Journal of Econometrics 58, 223-256.

Shalev-Shwartz, S. and S. Ben-David (2014). Understanding Machine Learning: From Theory to Algorithms. New York, NY, USA: Cambridge University Press.

Syrganis, V. and M. Zampetakis (2020, July). Estimation and Inference with Trees and Forests in High Dimensions. arXiv e-prints, arXiv:2007.03210.

Tan, Z. (2020a). Model-assisted inference for treatment effects using regularized calibrated estimation with high-dimensional data. Annals of Statistics 48(2), 811-837.

Tan, Z. (2020b, March). Regularized calibrated estimation of propensity scores with model misspecification and high-dimensional data. Biometrika 107, 137-158.

van der Geer, S., P. Bühlmann, Y. Ritov, and R. Dezeure (2014). On asymptotically optimal confidence regions and tests for high-dimensional models. Annals of Statistics 42(3), 1166-1202.

Wager, S. and G. Walther (2015, March). Adaptive concentration of regression trees, with application to random forests. arXiv e-prints, arXiv:1503.06388.

$\mathrm{Zhu}, \mathrm{Y}$. (2017). Nonasymptotic analysis of semiparametric regression models with highdimensional parametric coefficients. The Annals of Statistics 45(5), 2274-2298.

Zhu, Y., Z. Yu, and G. Cheng (2019). High-dimensional inference in partially linear models. Volume 89, pp. 2760-2769.

Zimmert, M. and M. Lechner (2019, August). Nonparametric estimation of causal heterogeneity under high-dimensional confounding. arXiv e-prints, arXiv:1908.08779. 